\title{
Bunje (Novo Selo, Croatie), campagnes 2017-2018
}

Emmanuel Botte, Audrey Bertrand, Kristina Jelinčić, Nicolas Leys et Antoine Boisson

\section{(2) OpenEdition \\ Journals}

\section{Édition électronique}

URL : https://journals.openedition.org/cefr/2419

DOI : $10.4000 /$ cefr. 2419

ISSN : 2282-5703

Éditeur

École française de Rome

Référence électronique

Emmanuel Botte, Audrey Bertrand, Kristina Jelinčić, Nicolas Leys et Antoine Boisson, « Bunje (Novo Selo, Croatie), campagnes 2017-2018 ", Chronique des activités archéologiques de l'École française de Rome [En ligne], Balkans, mis en ligne le 23 janvier 2019, consulté le 21 septembre 2021. URL : http:// journals.openedition.org/cefr/2419; DOI : https://doi.org/10.4000/cefr.2419

Ce document a été généré automatiquement le 21 septembre 2021.

(c) École française de Rome 


\title{
Bunje (Novo Selo, Croatie), campagnes 2017-2018
}

\author{
Emmanuel Botte, Audrey Bertrand, Kristina Jelinčić, Nicolas Leys et \\ Antoine Boisson
}

\section{NOTE DE L'AUTEUR}

Avec la collaboration de Léo Cagnard (université Montpellier 3) ; Jere Drpić (Musée de Varaždin) ; David Ilić (université de Zagreb) ; Ivana Ožanić (Institut archéologique de Zagreb) ; Alexia Rozak

\section{Introduction}

1 Le chantier de Bunje sur l'île de Brač constitue la première étape d'un programme portant sur les villae de l'Adriatique orientale, conduit par E. Botte (centre CNRS), A. Bertrand (université Paris-Est Marne-la-Vallée) et K. Jelinčić (Institut Archéologique de Zagreb) et intitulé « Recherches sur l'exploitation économique de la Dalmatie à l'époque romaine (II s. av. J.-C. - $\mathrm{II}^{\mathrm{e}} \mathrm{s}$. apr. J.-C.) ». L'intention des auteurs de ce projet est de mesurer, à travers des opérations de fouilles et de prospections, l'impact économique de la présence romaine en Dalmatie.

2 La première campagne menée en 2015 sur le site de Bunje (commune de Novo Selo) sur l'île de Brač était destinée à obtenir une vision d'ensemble de l'ampleur du site et de l'état de préservation de ses vestiges. Il en était ressorti un site assez étendu (les vestiges couvrant un espace de près de $15000 \mathrm{~m}^{2}$, et la villa couvrant à l'intérieur de celui-ci près de $2500 \mathrm{~m}^{2}$ ) et dont les structures sont dans l'ensemble assez bien conservées, ce qui confirme les observations effectuées lors d'un premier rapport mené par K. Jelinčić en $2012^{1}$. Le site est inscrit au registre des monuments culturels sous le numéro 486, et protégé en tant que tel auprès de la Surintendance de Split par décision du 26 septembre 1979 (sous le numéro 17/41-1979). 
Les opérations menées sur ce site s'inscrivent dans une recherche plus large visant à clarifier les mécanismes et la chronologie de l'occupation du territoire de la Dalmatie centrale en amont et en aval de la conquête romaine à la fin de la période tardorépublicaine. Au sein de cette problématique, les fouilles de la villa de Bunje nous permettront de déterminer le cadre historique et environnemental qui accueille cette structure, ainsi que l'organisation de sa production (fig. 1).

Fig. 1 - Carte de situation de l'île de Brač et de la commune de Novo Selo.

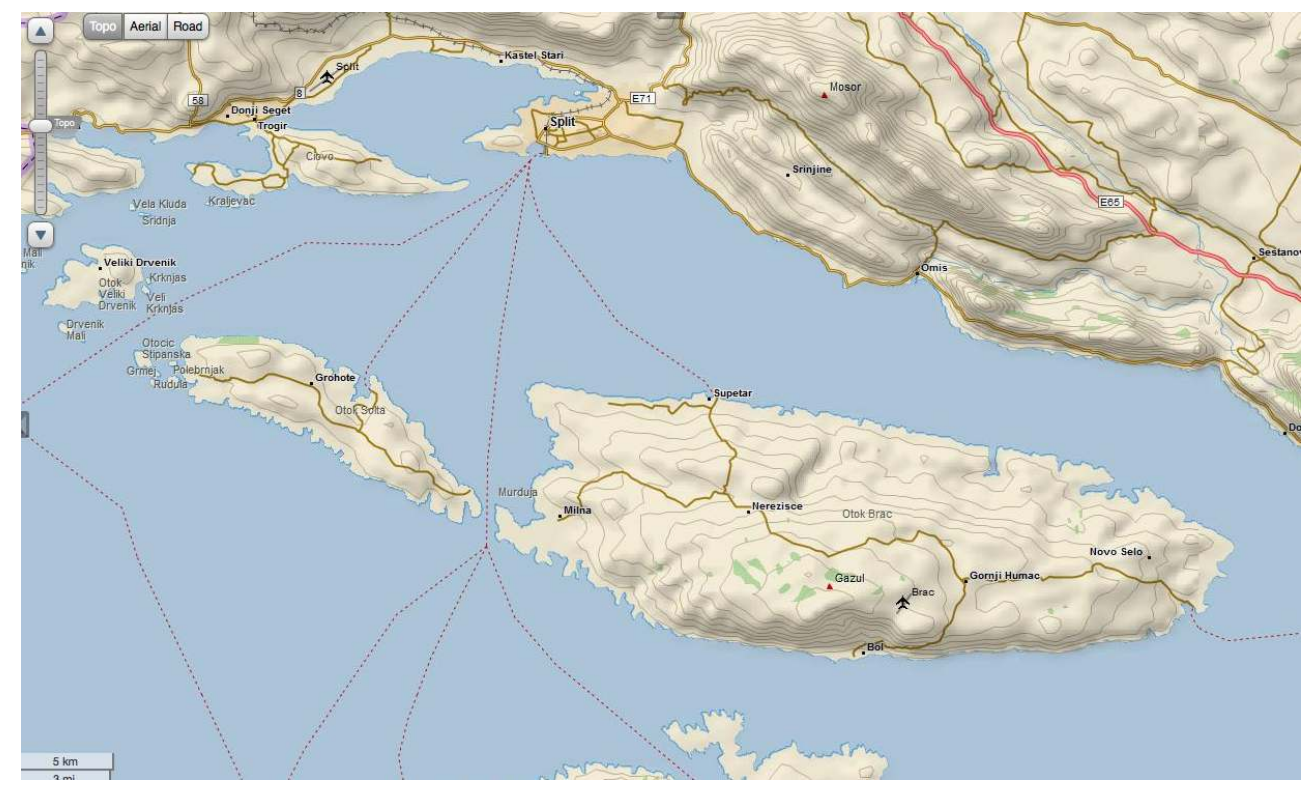

(c) Googlemaps.

En 2016, la campagne nous avait permis de faire des avancées significatives dans la compréhension du plan de la villa, en faisant apparaître la partie résidentielle (pars urbana) et la partie productive (pars rustica) de part et d'autre part d'une grande cour centrale. Nous avions notamment mis au jour les thermes de la villa dans la partie sudouest, tandis que dans la partie productive les premiers espaces dédiés à la production d'huile d'olive avaient été appréhendés. Durant cette campagne, nous avions également pu découvrir les limites occidentale et méridionale de la villa.

La campagne d'avril 2017 était destinée à mettre au jour l'ensemble de la partie résidentielle de la villa, notamment dans sa partie septentrionale, tout en cherchant les traces possibles des premiers états de la villa. Nos vœux n'ont été que partiellement exaucés car si nous avons pu effectivement découvrir le plan de la pars urbana au nord des thermes, son état de conservation très dégradé n'a livré pratiquement aucune stratigraphie, nous privant alors d'un phasage historique de l'occupation de cette partie du site. Néanmoins, la poursuite des dégagements à l'est des thermes, en direction de la cour centrale, nous a apporté des résultats plus satisfaisants. Ils nous ont notamment permis d'intercepter des niveaux d'occupation passant sous les murs de la villa telle qu'elle est actuellement visible, et comportant du mobilier qui nous a permis de confirmer qu'il existait bien une première installation datable de la seconde moitié du $\mathrm{I}^{\mathrm{er}}$ siècle de notre ère, sans doute de l'époque flavienne. 


\section{Déroulement et objectifs des campagnes d'octobre 2017 et avril 2018}

6 Les campagnes de fouilles se sont respectivement tenues du 9 au 20 octobre 2017, et du 16 avril au 3 mai 2018, sous la direction d'E. Botte, A. Bertrand et K. Jelinčić Vučković. L'équipe de fouille était constituée d'A. Boisson, L. Cagnard (université Montpellier 3), J. Drpić (Musée de Varaždin), D. Ilić (université Zagreb), N. Leys (université Paris 4), I. Ožanić (Institut archéologique de Zagreb) et A. Rozak.

7 Les campagnes ont été financées par l'École française de Rome, le ministère de l'Europe et des Affaires étrangères, le Centre Camille Jullian, le ministère de la Culture de Croatie et la municipalité de Selca. Les mobiliers en cours d'étude et ceux considérés comme importants pour la datation et l'histoire du site ont été consignés à l'Institut archéologique de Zagreb. L'étude archéozoologique est réalisée par Siniša Radović (Académie des sciences et des arts à Zagreb); l'étude anthracologique par Christophe Vaschalde (université Montpellier 3); l'étude paléobotanique par Margaux Tillier (université Montpellier 3); l'étude micro-morphologique des sols par Cristiano Nicosia (université de Padoue); l'étude du verre par Bartul Siljeg (Institut archéologique de Zagreb); les études anthropologiques par Mario Novak (Institut d'anthropologie de Zagreb) ; les études numismatiques par Anja Bertol (université de Zagreb); les analyses chimiques par Nicolas Garnier (Laboratoire Nicolas Garnier). N. Leys, responsable de la photogrammétrie sur le site, a fait une modélisation 3D du site et des principaux blocs de pierre. Les relevés topographiques et les plans ont été réalisés par K. Jelinčić Vučković. L'étude du mobilier céramique a été réalisée par $\mathrm{E}$. Botte et les dessins par A. Boisson.

8 Les objectifs de ces missions étaient concentrés respectivement sur la fin de la partie résidentielle de la villa (octobre 2017) et sur sa partie productive (avril-mai 2018). Nous souhaitions en effet découvrir la suite de l'organisation des espaces découverts en 2016 par rapport au reste de la pars urbana, en dégageant notamment l'ensemble du secteur 5 et observer son articulation avec le secteur 6 situé de l'autre côté de la cour centrale. Ainsi il était envisagé de relier ces deux secteurs pour en comprendre la disposition par rapport à la cour centrale, mais également d'ouvrir un sondage au sud du grand mur méridional de la villa (MR 6009) pour comprendre l'articulation entre cette dernière d'une part et le grand sarcophage et l'espace dans lequel il était installé d'autre part.

Du côté de la pars rustica (secteurs 6 et 8), les objectifs étaient à la fois de comprendre son organisation mais également d'être en mesure d'identifier les productions réalisées et de pouvoir commencer à les replacer dans le phasage général de la villa.

Une grande partie du travail de ces campagnes a consisté à démanteler les murs modernes à l'aide d'une pelle mécanique mise à disposition par la mairie de Selca. En effet, le site ayant fait l'objet de cultures agricoles depuis près d'un siècle, les différentes générations ont constitué sur le site de grands amas de pierres (gomila) pouvant atteindre près de $2 \mathrm{~m}$ de hauteur, et qui gênaient considérablement la lecture du site dans son ensemble. Bien entendu une grande partie de ces amas de pierres et du mobilier archéologique qui s'y trouve correspond au démantèlement des structures archéologiques de la villa antique. De ce fait, le démontage de ces structures est réalisé systématiquement en présence de plusieurs membres de l'équipe, le mobilier conservé et 
les pierres travaillées mises de côté en vue de la restauration du site et de sa présentation au public lorsque les fouilles seront achevées (fig. 2-3).

Fig. 2 - Vue du site par drone au début de la campagne.

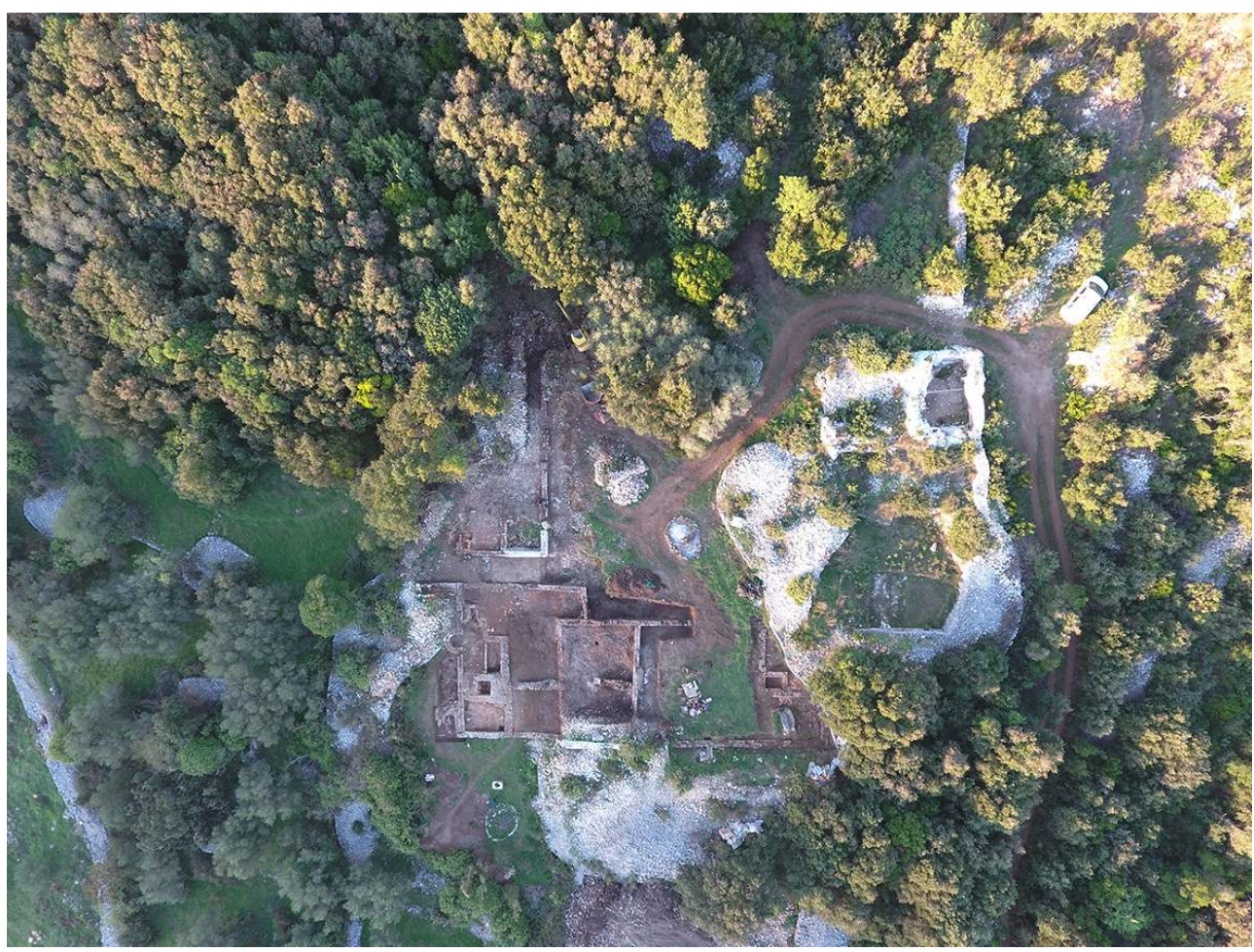

Cl. E. Botte 
Fig. 3 - Plan de la villa avec les secteurs fouillés (situation avant la fouille).

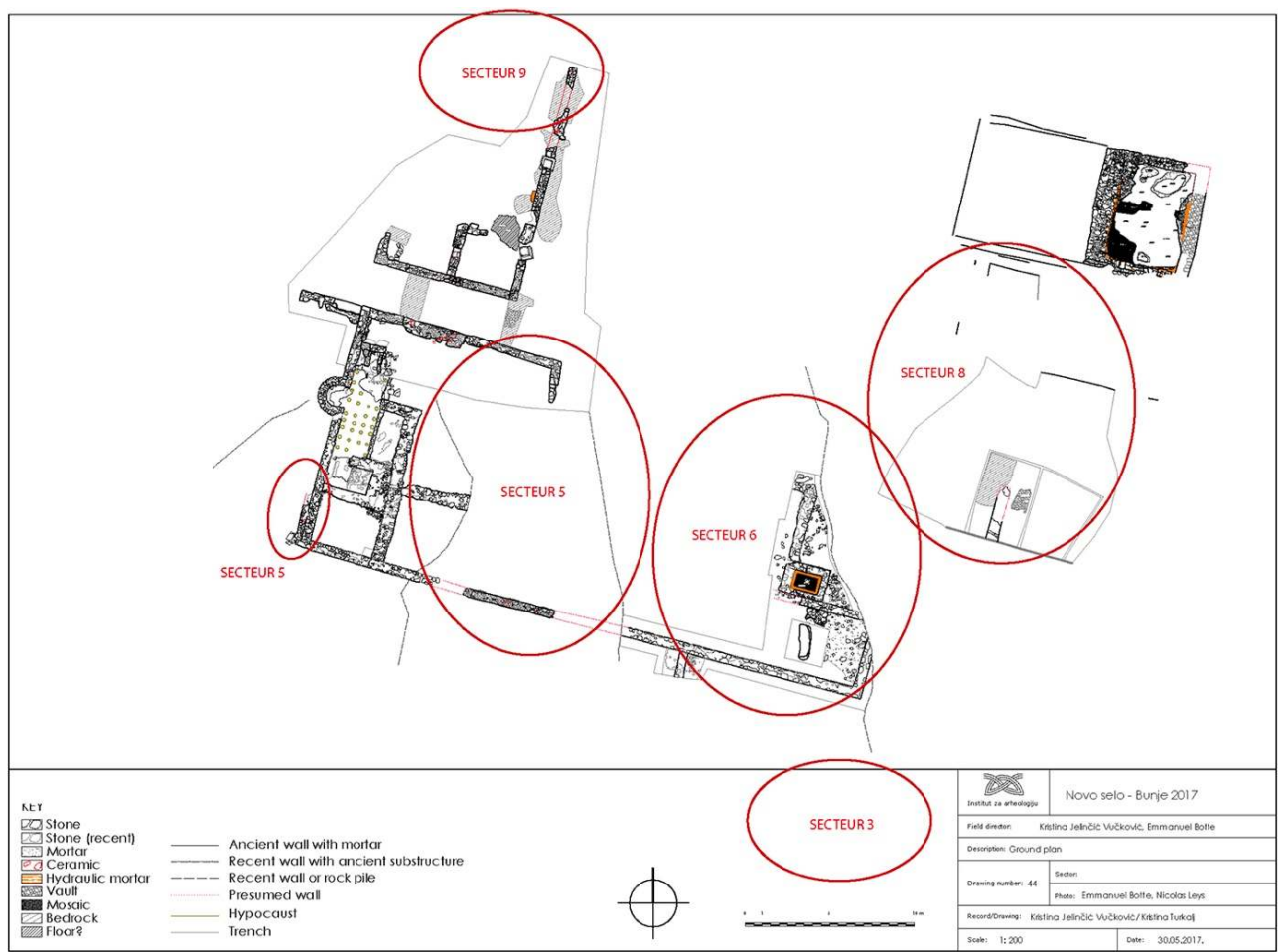

K. Jelinčić.

\section{Résultats}

\section{Secteur 3}

11 Le secteur 3, situé au sud du grand mur de limite méridionale de la villa (MR 6009), n'avait pas encore fait l'objet d'une exploration poussée. Nous l'avions créé en 2015 car s'y trouve un sarcophage, largement pillé et exposé aux intempéries depuis près d'un siècle, que sa typologie permet de dater $\mathrm{du} \mathrm{V}^{\mathrm{e}}$ siècle de notre ère ${ }^{2}$. Il appartient à la catégorie des sarcophages que l'on trouve en grande quantité sur le site de Salona, et dont on sait désormais qu'ils provenaient en grande partie des carrières de l'île de Brac. Nous avons décidé cette année d'effectuer un sondage autour de cette structure pour déterminer son implantation par rapport à la villa romaine (fig. 4). 
Fig. 4 - Indication des principales unités stratigraphiques du Secteur 3.

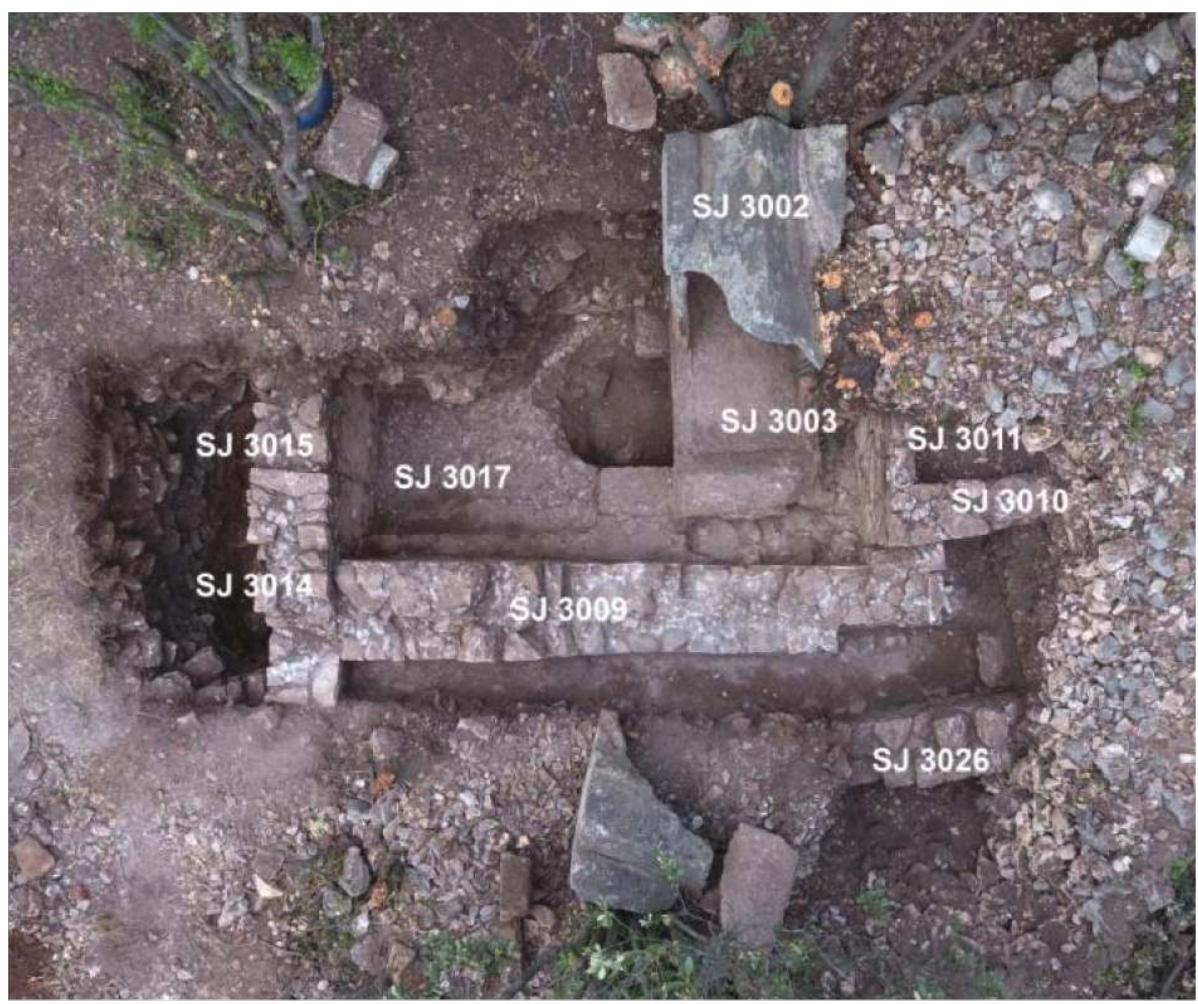

Cl. E. Botte et K. Jelinčić

Le sarcophage était pris dans une épaisse végétation dans laquelle était surtout visible l'acrotère de l'angle nord-ouest du couvercle, portant un monogramme chrétien (fig. 5). Dans cette végétation nous avons pu mettre au jour un fragment du couvercle du sarcophage (situé au sud de la cuve) portant une croix gravée sur la face sud mais dans un mauvais état de conservation. On observe un exemple identique sur l'acrotère du sarcophage découvert à Trišćenik, au sud-est de Dračevica³ ${ }^{3}$ Bulić écrit en 1914 qu'il y avait un autre acrotère portant un monogramme chrétien qui aurait été transporté au Musée archéologique de Split ( $n^{\circ} 439$, CAT D), mais celui-ci est aujourd'hui introuvable ${ }^{4}$. 
Fig. 5 - Monogramme chrétien sur l'acrotère nord-est du couvercle du sarcophage 3002.

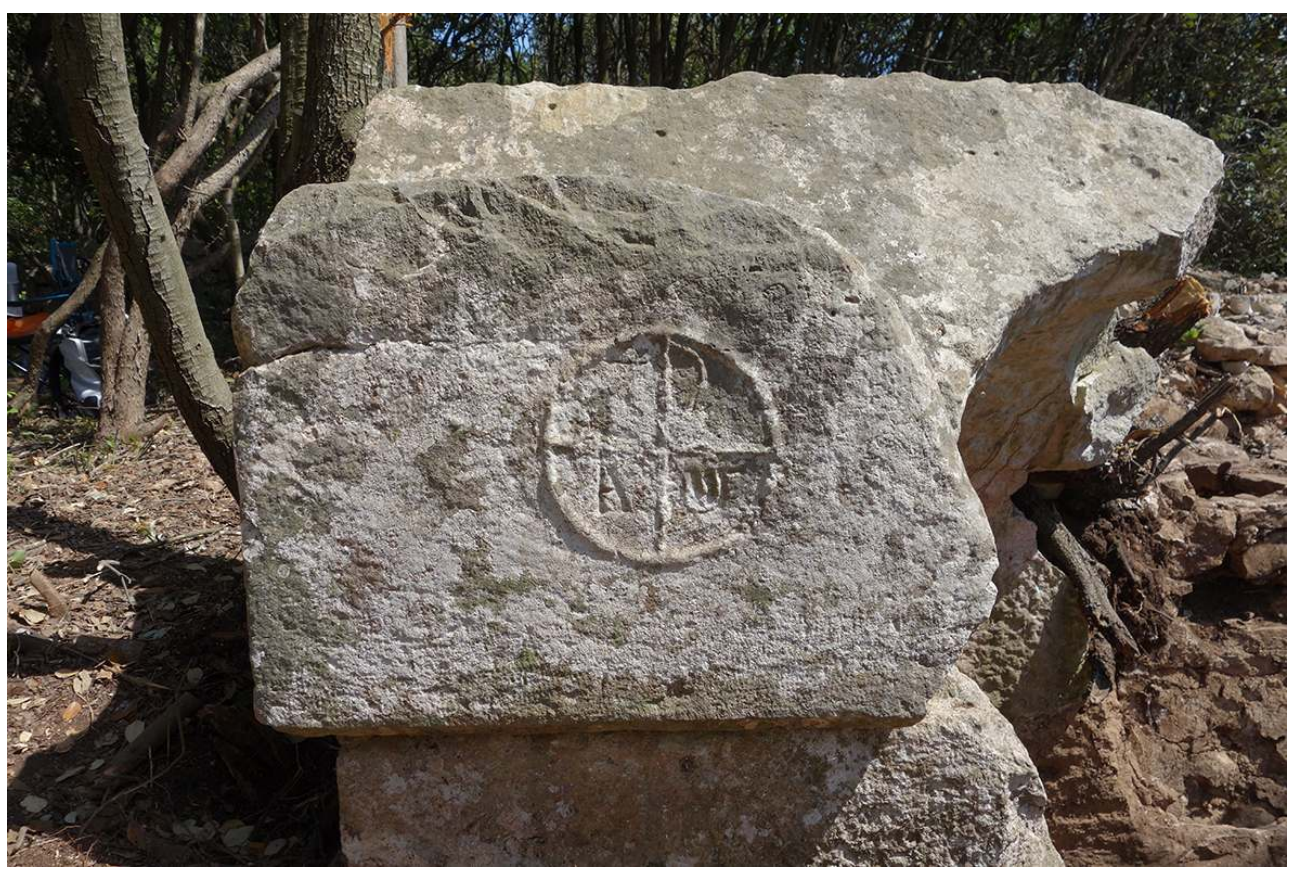

Cl. E. Botte

Le sondage réalisé autour du sarcophage nous permet de proposer un phasage général du secteur 3. Il apparaît ainsi qu'il existait un espace contemporain de la villa romaine des $\mathrm{II}^{\mathrm{e}}$ -III siècles de notre ère, mais pour lequel nous ne disposons pas d'éléments suffisants pour en déterminer la fonction. Situé à l'extérieur de la villa, rien n'empêche de considérer qu'il ait pu s'agir de dépendances, ou d'un espace qui aurait déjà eu une vocation funéraire. On peut dater de cette période les murs MR 3009 et 3014. Il existait à cette période une ouverture dans le mur MR 3014, qui sera plus tard bouchée par le mur MR 3015.

C'est dans cet espace qu'est installée la cuve du sarcophage 3003. Sans doute pour éviter un affaissement du terrain en raison de son poids, un niveau de grands blocs de calcaire a été disposé comme lit de pose pour recevoir la cuve (US 3019) (fig. 6). L'ensemble de la pièce est alors doté d'un sol en béton (SL 3017) reposant sur le ressaut de fondation des murs MR 3009 et 3014 et venant au contact des blocs du lit de pose 3019 et de la cuve du sarcophage 3003 (fig. 7). 
Fig. 6 - Vue depuis le sud-ouest des blocs (US 3019) du lit de pose de la cuve du sarcophage 3003.

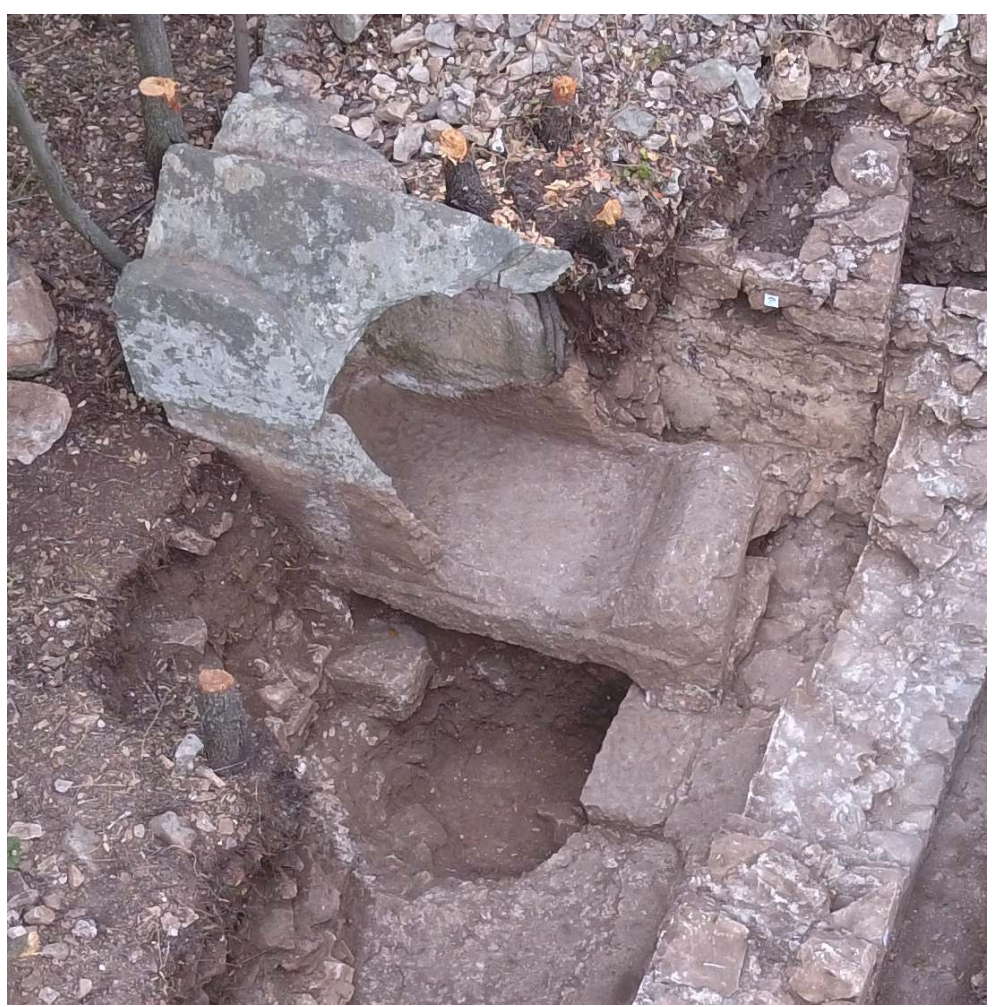

Cl. E. Botte.

Fig. 7 - Vue depuis l'est du sol SL 3017 dans la pièce où se trouvait le sarcophage.

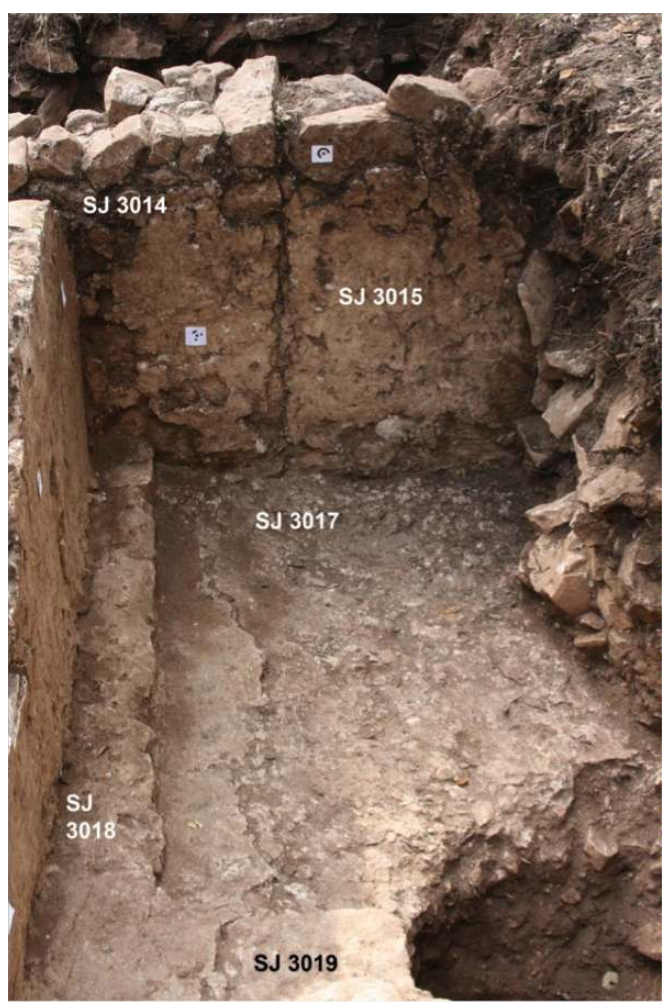

Cl. K. Jelinčić. 
15 À une période que l'on ne peut fixer au plus tôt qu'au cours du $\mathrm{V}^{\mathrm{e}}$ siècle, mais après l'installation du sarcophage, le bouchage MR 3015 est construit au nord du mur MR 3014, indiquant que l'accès à cet espace s'effectuait par une autre ouverture. Les murs MR 3010 et 3011 sont alors construits à l'angle nord-est du mur MR 3009. Il est en tout cas clair que cet espace est toujours fréquenté, car un enduit de bonne qualité est appliqué sur l'ensemble des murs cités, créant ainsi un ensemble homogène. C'est dans ces niveaux contemporains de réaménagements que nous avons mis au jour un contorniate à l'effigie de Trajan, médaillon commémoratif frappé dans un atelier privé au $\mathrm{IV}^{\mathrm{e}}$ ou $\mathrm{V}^{\mathrm{e}}$ siècle ${ }^{5}($ fig. 8-9).

Fig. 8 - Avers du contorniate à l'effigie de Trajan.

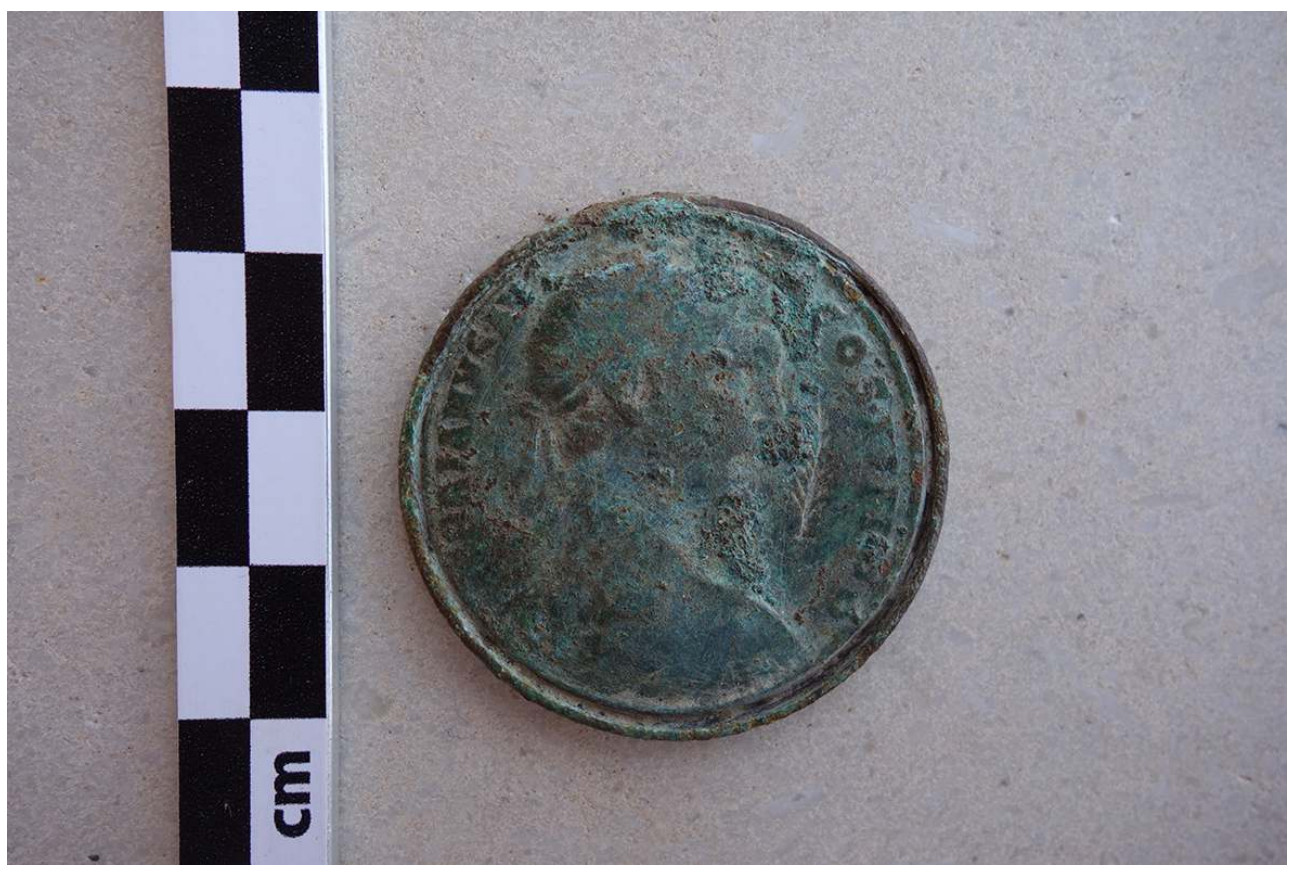

Cl. E. Botte 
Fig. 9 - Revers du contorniate à l'effigie de Trajan.

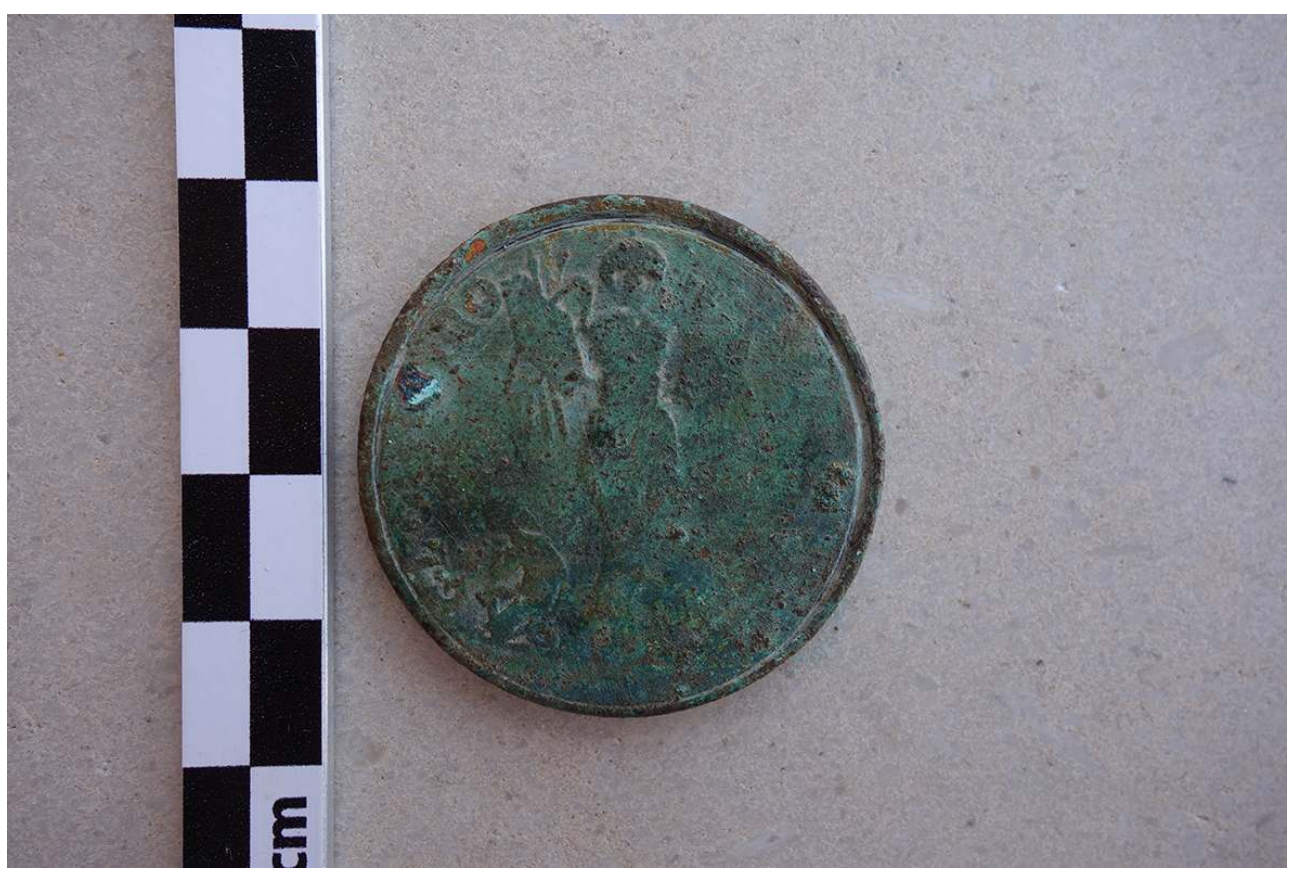

Cl. E. Botte.

\section{Secteur 5}

16 Les opérations dans le secteur 5 étaient ciblées sur deux zones. D'une part, nous souhaitions identifier la structure apparaissant à l'ouest du mur des thermes MR 5003 ; d'autre part nous souhaitions observer la nature des vestiges à l'est des thermes, afin de comprendre l'articulation avec la cour centrale et par conséquent la pars rustica de la villa.

Contre le parement ouest du mur MR 5003 était visible depuis les premiers relevés du site effectués en 2015 un enduit en béton de tuileau dont la destination n'était pas assurée. Nous avons donc ouvert un sondage depuis l'angle sud-ouest de la villa (angle des murs MR 5001 et 5003) jusqu'au parement externe de l'abside ABS 5011. Il en est sorti une seconde abside des thermes, mais dans un état de conservation assez déplorable ( fig. 10-11). On peut néanmoins établir que l'on y accédait par une marche dans l'angle nord-est dont l'arrachement est encore visible, et que ce bassin, comme l'ensemble des bassins recevant des liquides, que ce soit pour le stockage (citernes), le loisir (bassins des thermes) ou la production (bassins de récolte de l'huile et du vin, fouloirs), était équipé d'un sol en mosaïque. La raison de ce mauvais état de conservation est due au fait que ce bassin a été perforé durant l'Antiquité tardive par l'installation d'une tombe en inhumation. Cette sépulture ne contenait pas, ou plus car elle était très endommagée, de mobilier datant, hormis une boucle de ceinture. L'étude anthropologique de Mario Novak a révélé que la sépulture contenait en fait 4 individus, puisque l'étude a mis en évidence qu'il y avait 4 fragments de cuisse droite et 4 os pelviens droits. Leur étude a démontré que dans la sépulture se trouvaient 4 enfants, l'un âgé de 6 mois, deux d'environ 1 an et demi à 2 ans, et un dernier de 3 à 4 ans (fig. 12-13). 
Fig. 10 - Vue de l'abside ABS 5074 du secteur des thermes (secteur 5).

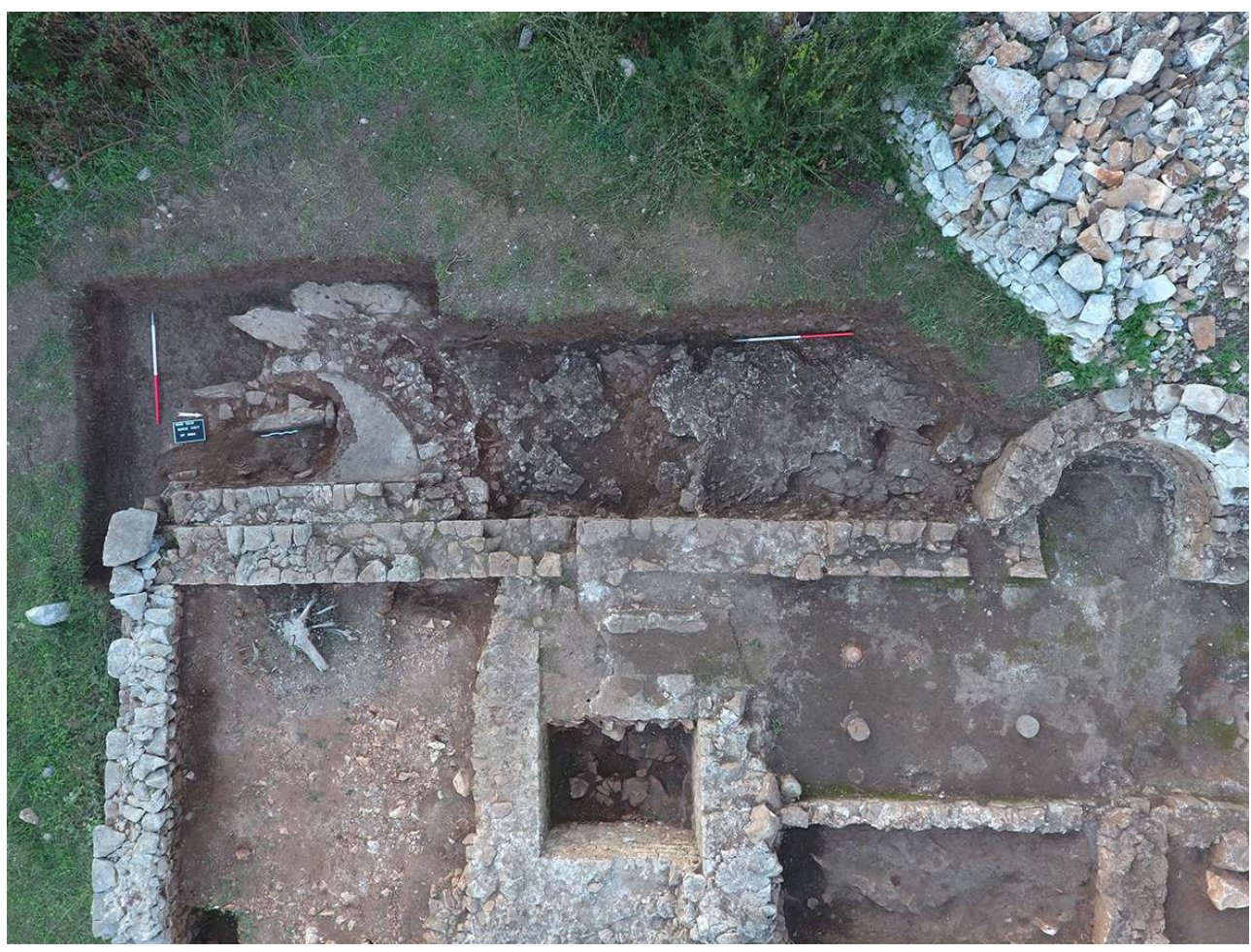

Cl. E. Botte

Fig. 11 - Vue depuis l'ouest de l'abside ABS 5074 du secteur des thermes (secteur 5).

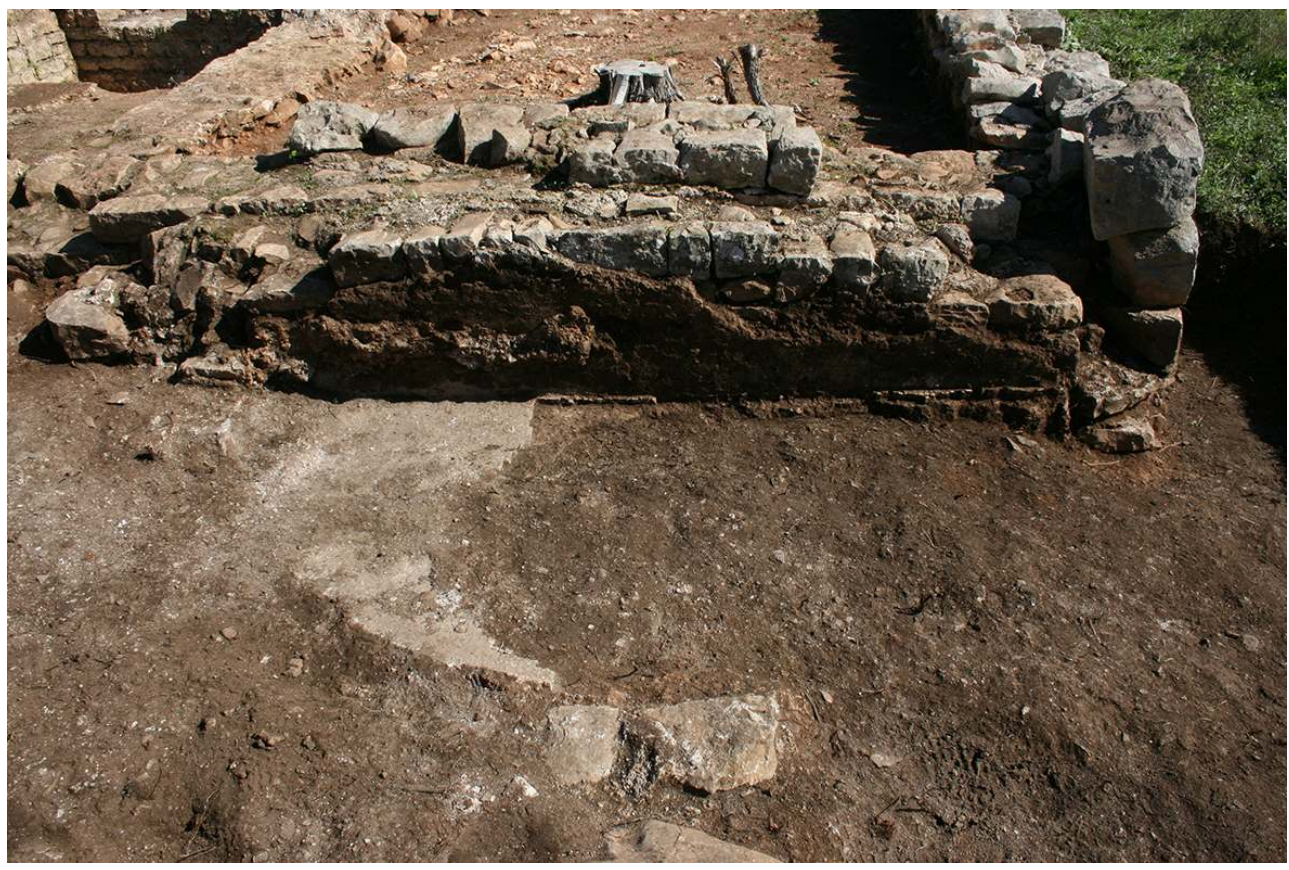

Cl. E. Botte. 
Fig. 12 - Vue du premier squelette (SQ 5096) de la sépulture SP 5084 du secteur des thermes (secteur 5).

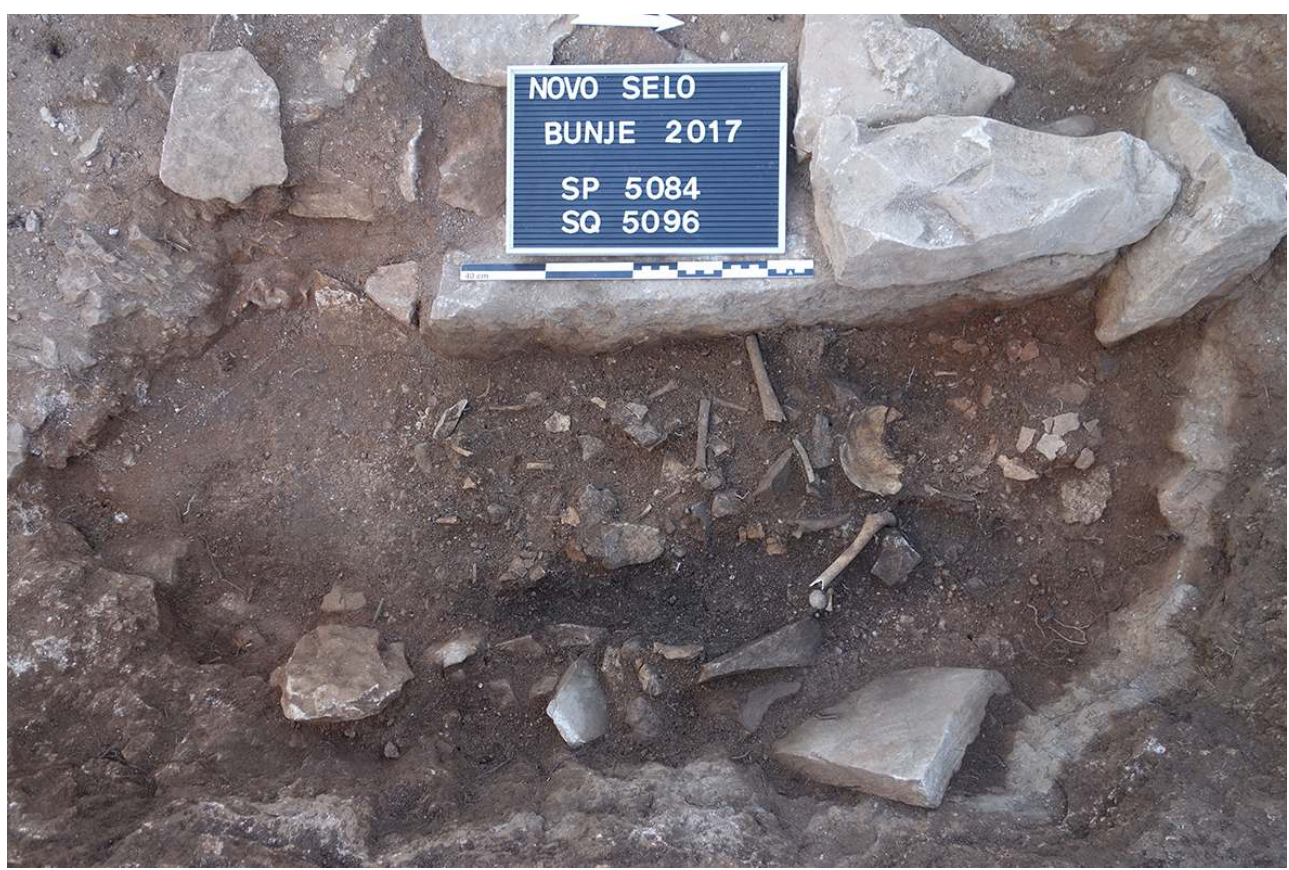

Cl. E. Botte.

Fig. 13 - Vue du second squelette (SQ 5106) de la sépulture SP 5084 du secteur des thermes (secteur 5).

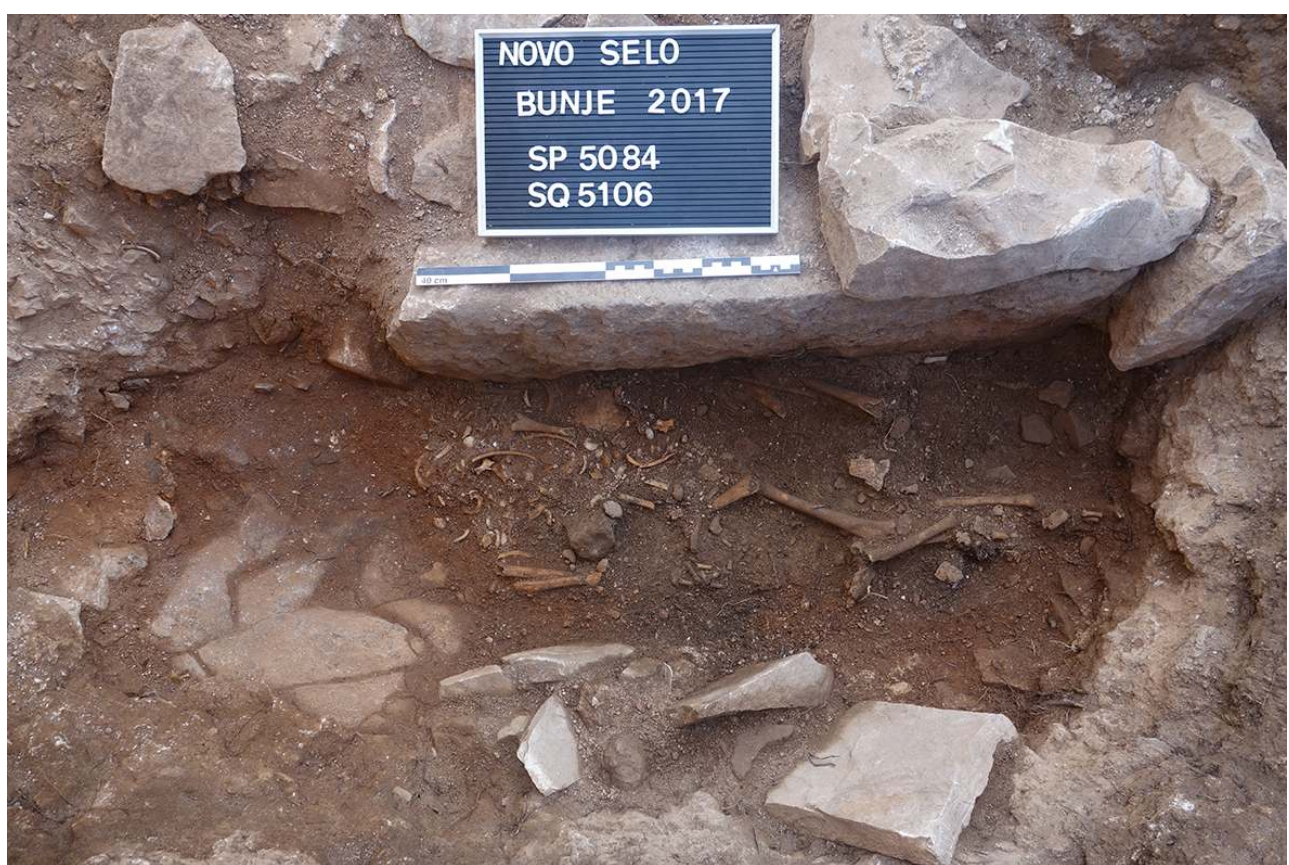

Cl. E. Botte.

Les autres travaux menés dans le secteur 5 concernaient la partie située à l'est des thermes dégagés en 2016. Les opérations ont surtout consisté à enlever à l'aide d'une pelle mécanique l'énorme amas de pierres qui atteignait près de deux mètres de hauteur 
par endroits. Il en est sorti un grand espace de 10,6 m sur 6,8 m, doté d'un pilier central ( fig. 14), avec une porte bouchée sur son côté nord. Le dégagement de ce secteur nous a également permis de découvrir un décroché dans lequel se trouve un passage également bouché (US 5057), qui doit constituer l'entrée dans l'espace thermal (fig. 15).

Fig. 14 - Vue de la pièce à pilier située à l'est des thermes.

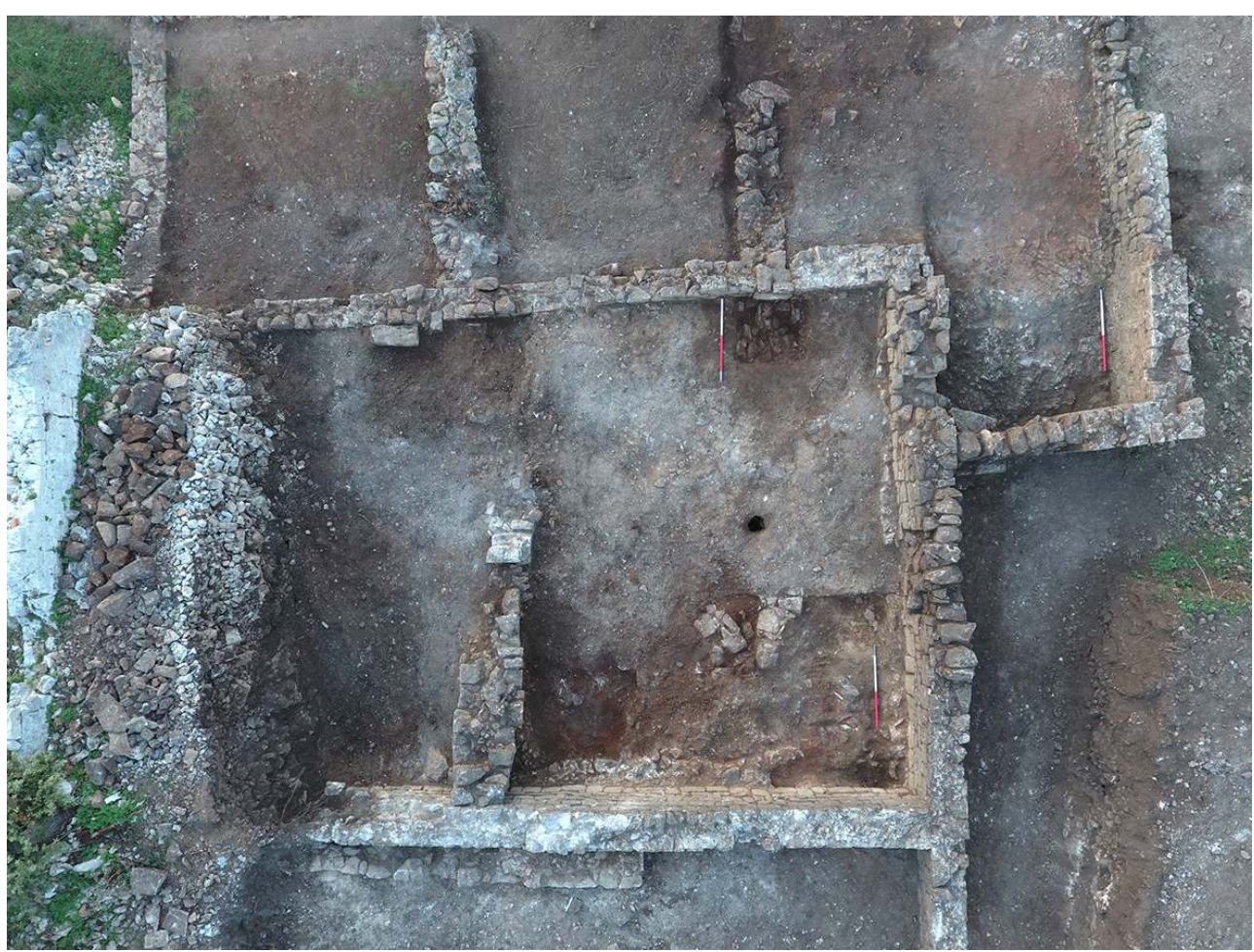

Cl. E. Botte. 
Fig. 15 - Vue depuis l'ouest du passage bouché (US 5057) donnant accès à l'espace thermal.

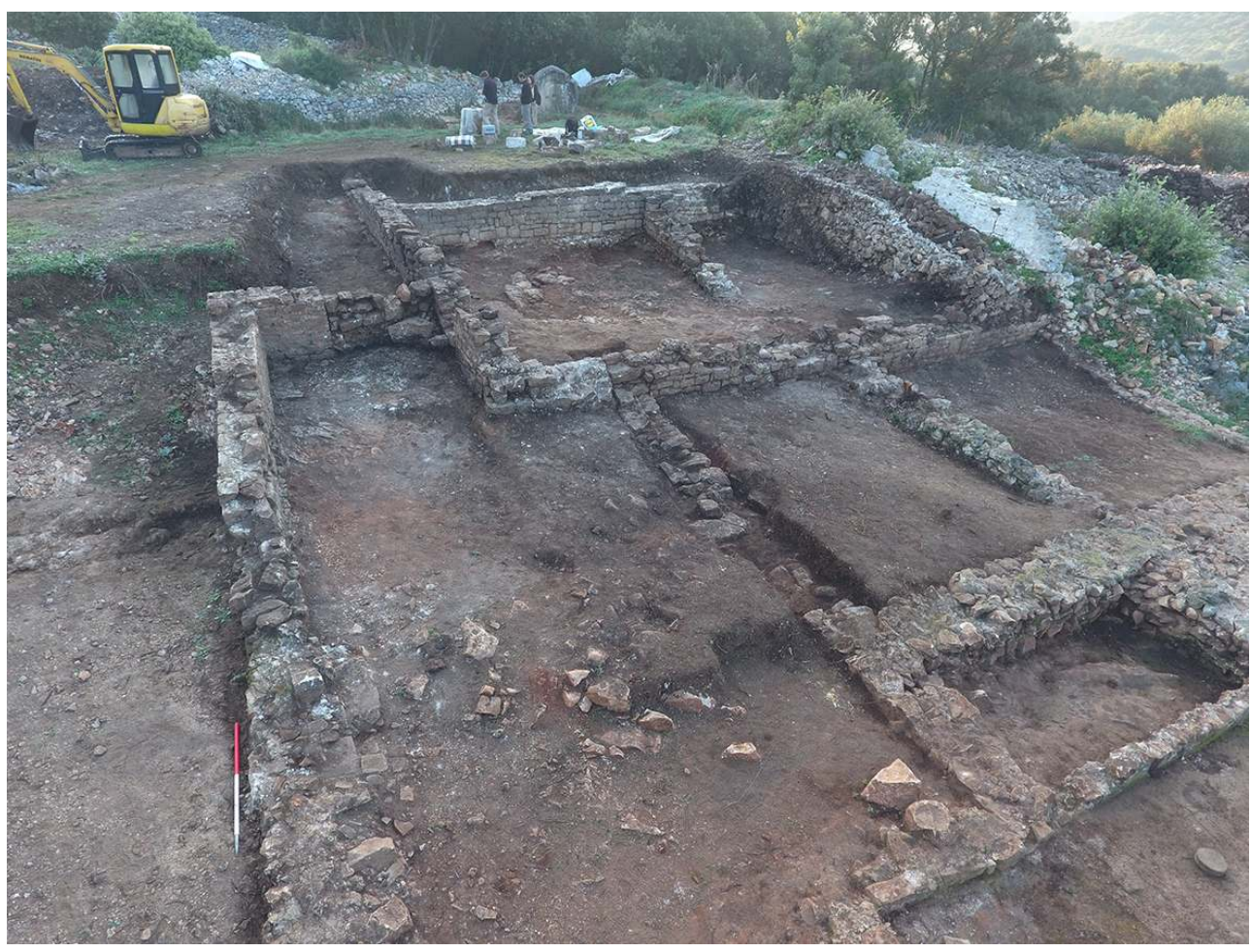

Cl. E. Botte.

19 Dans la pièce équipée d'un pilier central, la fouille commencée en octobre 2017 et poursuivie en avril 2018 a permis de révéler des niveaux archéologiques passant sous les murs de la villa telle qu'elle est actuellement visible, et sous ces niveaux se trouvaient des murs plus anciens (fig. 16). C'est une donnée très importante pour l'histoire du site car jusqu'à présent, même si du mobilier ancien apparaissait de manière résiduelle, nous n'étions pas en mesure de projeter la nature de cette occupation antérieure. Grâce aux murs qui ont été découverts, et au mobilier qui les recouvrait, nous sommes désormais en mesure de proposer l'existence d'une première installation, bien plus modeste dans ses dimensions, sans doute construite durant l'époque flavienne. Le mobilier découvert dans les niveaux recouvrant ces murs arasés est datable des premières décennies du $\mathrm{II}^{\mathrm{e}}$ siècle de notre ère, notamment plusieurs types de sigillée africaine, ce qui nous permet de dater un grand remaniement et agrandissement du site dans le second tiers du $\mathrm{II}^{\mathrm{e}}$ siècle de notre ère. 
Fig. 16 - Vue des murs de la première phase d'occupation apparus dans la salle à pilier.

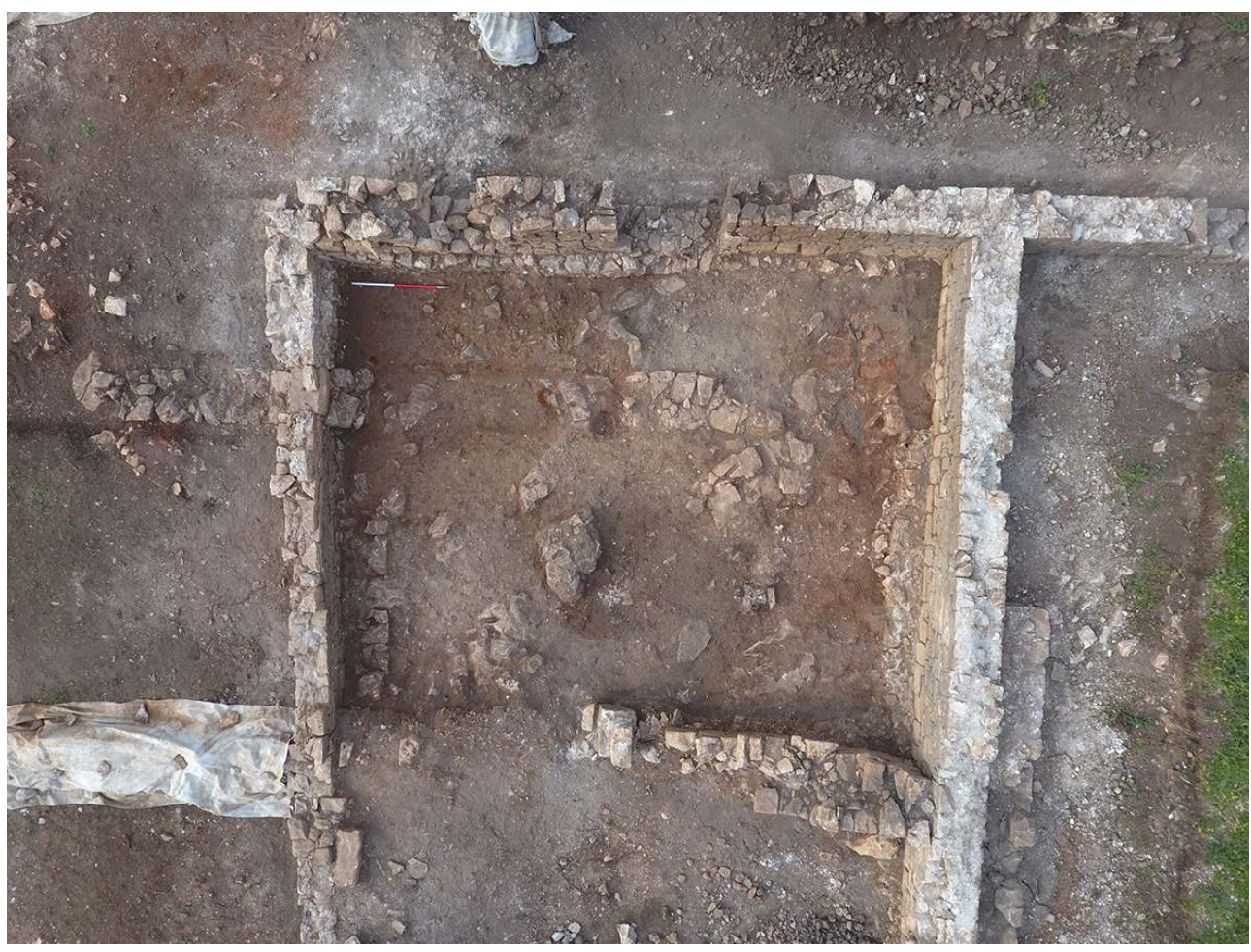

Cl. E. Botte.

\section{Secteur 6}

Les opérations dans le secteur 6 ont été brièvement entamées en octobre 2017 tandis qu'elles ont occupé une partie de l'équipe durant toute la campagne d'avril-mai 2018. En octobre 2017, les dégagements à la pelle mécanique des niveaux superficiels de pierres avaient permis d'apercevoir que le mur nord de la pièce à pilier continue vers l'ouest et donne sur une grande ouverture. Il faut donc en déduire que la cour centrale ne séparait pas complètement la pars urbana de la pars rustica mais que la villa était sans doute aménagée selon un plan en U. Ainsi sur tout le flanc sud de la villa se trouvaient d'ouest en est les thermes, puis un grand espace comportant le moulin destiné à écraser les olives avant de les emmener au pressoir. Nous chercherons à le vérifier durant les campagnes de 2019, mais il est probable que ce grand espace ait été destiné à recevoir les olives une fois récoltées et dans l'attente que leur processus de transformation ne débute.

21 En avril-mai 2018, nous avons consacré une partie de la campagne à ce secteur, qui nous a permis de grandement progresser sur la compréhension de l'organisation de la pars rustica. Cet espace, comme le secteur 8 , se trouvant lui aussi sous de grands tas de pierres comme les secteurs de la partie résidentielle de la villa, nous n'espérions pas obtenir beaucoup de données puisque dans ce dernier la stratigraphie était quasi inexistante. Il s'avère que dans cette partie de la villa, les pierres ont été jetées directement sur les structures antiques, sans que celles-ci aient été trop fortement récupérées ou détruites avant. Nous avons ainsi pu découvrir un grand fouloir à vin dans un état de conservation plutôt satisfaisant (fig. 17). 
Fig. 17 - Vue de l'espace du fouloir dans le secteur 6.

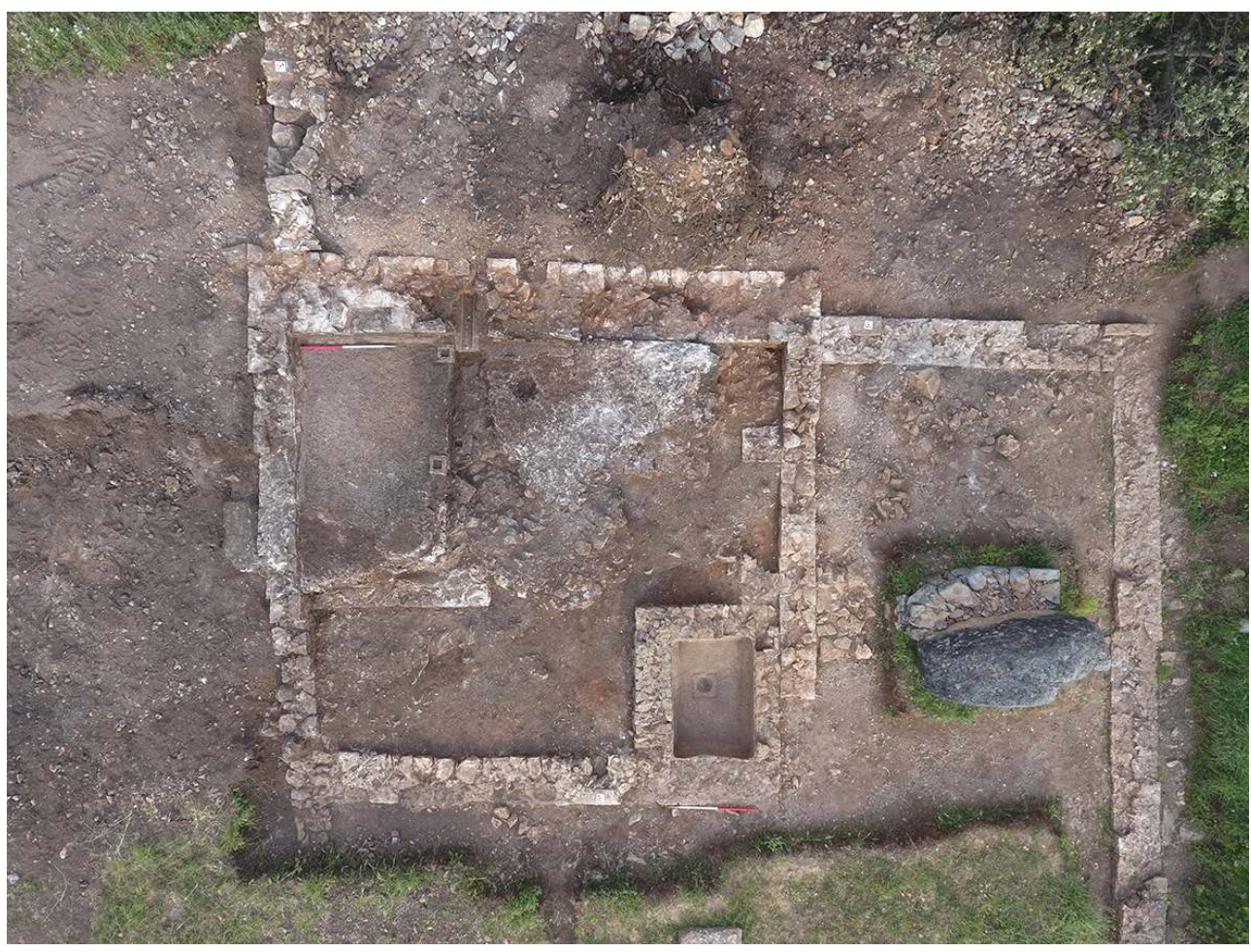

Cl. E. Botte.

22 Le fouloir est un grand espace mosaïqué de 3,80 m sur 3,30 m, qui était équipé en son centre d'une canalisation en calcaire qui menait le jus du foulage au pied dans un espace situé à l'ouest qui sera exploré durant la campagne d'octobre 2018. La partie nord du fouloir est très bien conservée tandis que sa partie sud a presque totalement disparu. Dans la partie nord, deux supports de poteaux en bois sont encore préservés (fig. 18), ce qui n'est pas sans rappeler les images de foulage qui nous sont parvenues sur décors de mosaïque. 
Fig. 18 - Vue depuis l'est du fouloir et de sa canalisation.

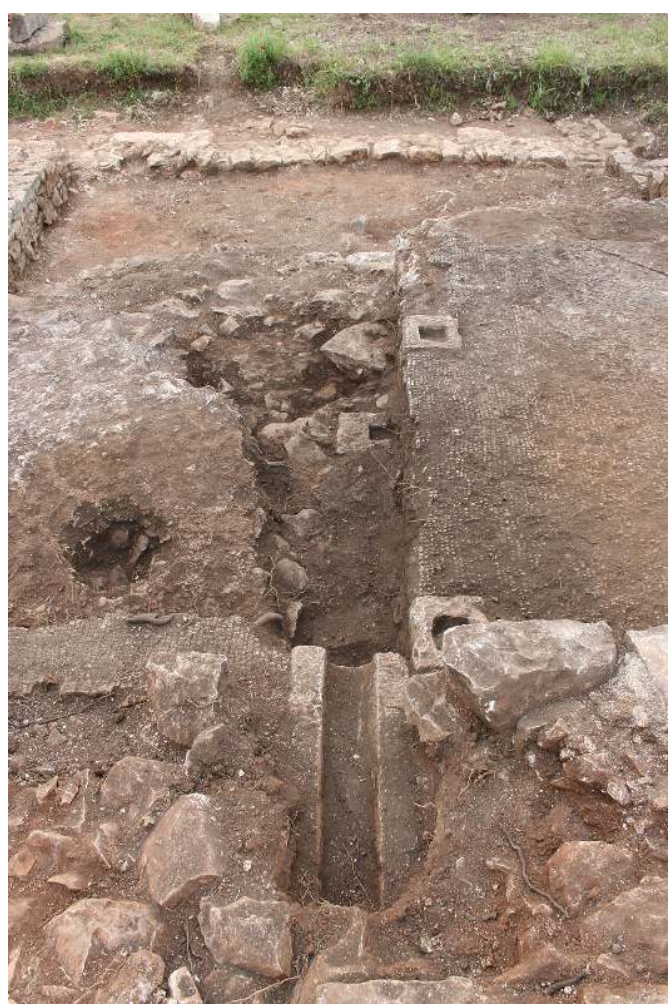

Cl. K. Jelinčić.

L'autre élément important dans ce secteur est qu'il apparaît en coupe qu'un bassin en béton de tuileau se trouve sous le fouloir, et qu'il était lui aussi équipé sur son côté sud de supports de poteau, ce qui nous incite à considérer qu'il s'agit d'un fouloir d'une première phase de la villa (fig. 19). Nous sommes ainsi tentés de considérer que le vin a constitué la première denrée produite sur le site, avant l'huile d'olive. Cela n'est pas surprenant pour plusieurs raisons: la première est que l'obtention d'un rendement avec la vigne est beaucoup plus rapide qu'avec l'olivier, de quelques années pour la première à au moins une décennie pour le second ; la seconde raison est qu'il s'agit d'un schéma que l'on peut observer en plusieurs points de la Méditerranée antique, notamment en Gaule et dans la péninsule Ibérique, où les premiers Romains venus s'installer dans de nouveaux territoires débutent par la vigne pour obtenir relativement rapidement une production fournissant un surplus vendable, tandis que les oliviers sont plantés dans la foulée mais sans que l'on puisse en attendre un rendement avant au moins une dizaine à une quinzaine d'années. 
Fig. 19 - Vue depuis l'ouest du fouloir en béton de tuileau antérieur au fouloir mosaïqué installé dans une seconde phase.

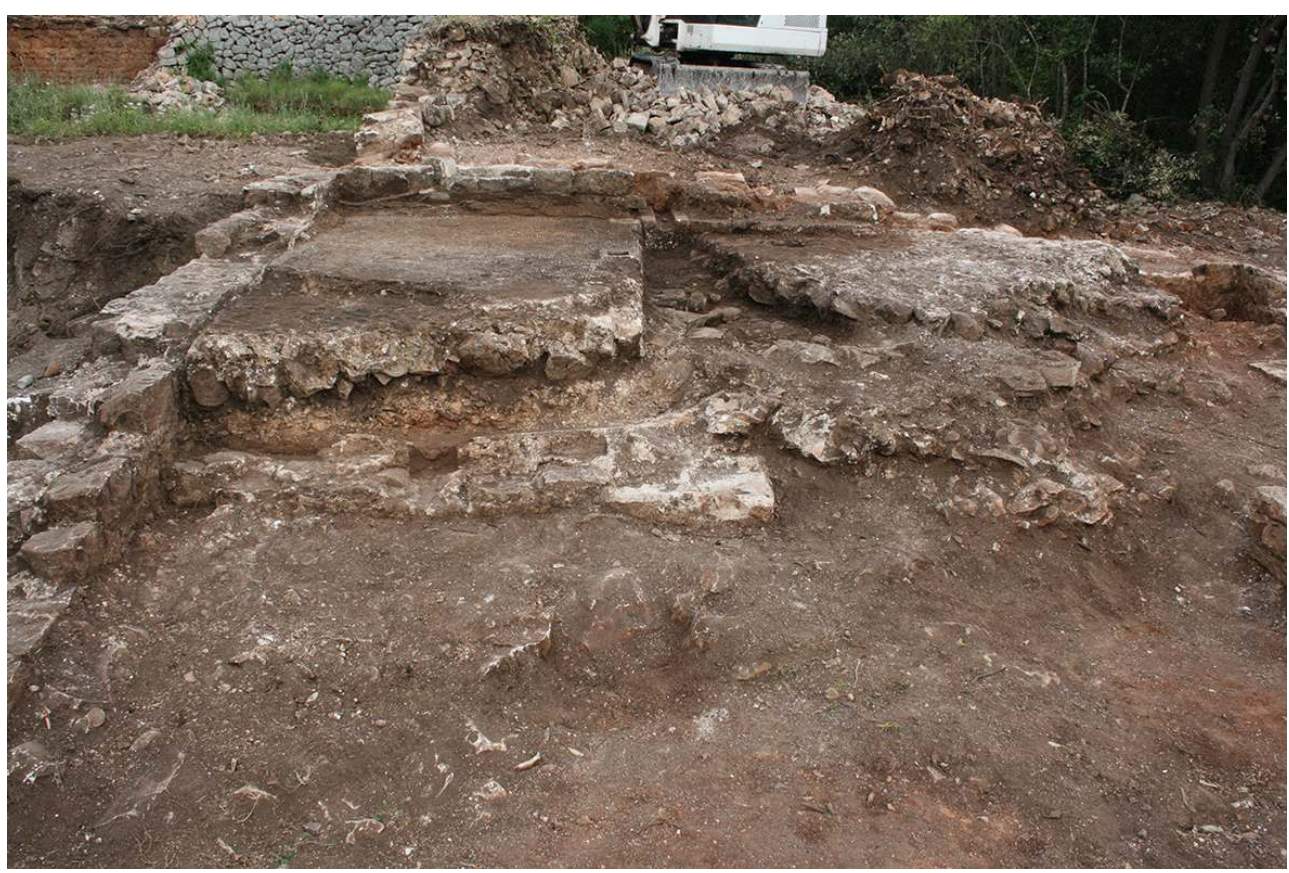

Cl. K. Jelinčić.

\section{Secteur 8}

Situé au nord du secteur 6 et contre la paroi sud des citernes, le secteur 8 a également livré des vestiges dans un état de conservation que nous ne soupçonnions pas. Tout d'abord à l'angle sud-ouest de la citerne occidentale (CT 10011) est apparu un bassin très endommagé en béton de tuileau, dont nous supposons qu'il a servi à recevoir l'eau des citernes. Dans les niveaux qui comblaient cette structure, nous avons mis au jour un basrelief représentant un phallus (fig. 20), ce qui n'est pas rare sur les sites de production : citons par exemple deux blocs au décor similaire découverts sur le grand site de production d'huile d'olive et d'amphores Dressel 6B de Loron en Istrie.

Fig. 20 - Bloc avec phallus en bas-relief découvert dans la pars rustica de la villa.

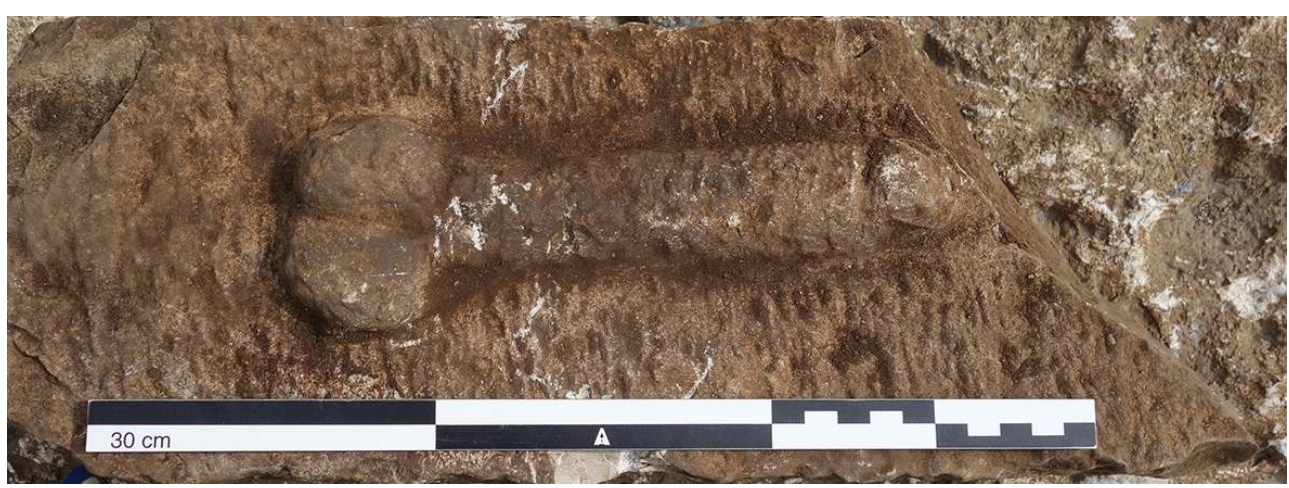

Cl. E. Botte. 
$\mathrm{Au}$ sud de ce bassin sont apparus les premières marches d'un escalier qui devait mener aux citernes. Seules trois marches sont conservées, mais leur emplacement ne nous incite pas à envisager d'autre hypothèse à l'heure actuelle.

Plus à l'est, directement contre la paroi méridionale de la citerne CT 10002, nous avons découvert un pressoir (PR 8048) dans un état de conservation remarquable (fig. 21). Il apparait clairement qu'il s'agit d'une dernière phase d'utilisation de cette structure car nous avons pu observer en coupe des montants en pierre d'un treuil de pressoir d'une phase antérieure (fig. 22). On peut donc voir d'est en ouest le bloc des arbores (fig. 23) qui recevait les jumelles encadrant le prelum, le mouton du pressoir ; vient ensuite la maie ( area) sur laquelle on peut très clairement observer les traces de regulae, les montants d'un coffre en bois dans lequel était jetée la pâte d'olives pour être pressée, système que l'on trouve sur d'autres sites de Dalmatie, notamment à Salone. Cette pratique en coffre de bois remplace ce qu'on trouve plus fréquemment, voire systématiquement, dans les autres provinces, à savoir les scourtins en sparterie qui étaient remplis de pâte d'olives et disposés sur la maie. À l'extrémité occidentale du complexe se trouve le bloc des stipites, qui soutenait les poteaux encadrant le mécanisme d'abaissement du levier du pressoir. Ce dernier état doit être relativement tardif car le bloc utilisé pour les stipites semble être le remploi d'un bloc d'arbores. Et les blocs que l'on voit dans la coupe appartiennent très clairement à des montants en calcaire encadrant un treuil permettant d'actionner le levier à l'aide de câbles. Ce système est attesté sur plusieurs sites d'Istrie et de Dalmatie, notamment à Salone dans des phases qui ne sont pas antérieures au $\mathrm{V}^{\mathrm{e}}$ siècle de notre ère. Cependant, rien n'empêche de penser que ce mécanisme ait été employé en Dalmatie auparavant.

Fig. 21 - Vue depuis le sud-ouest de l'ensemble du complexe du pressoir découvert en avril 2018.

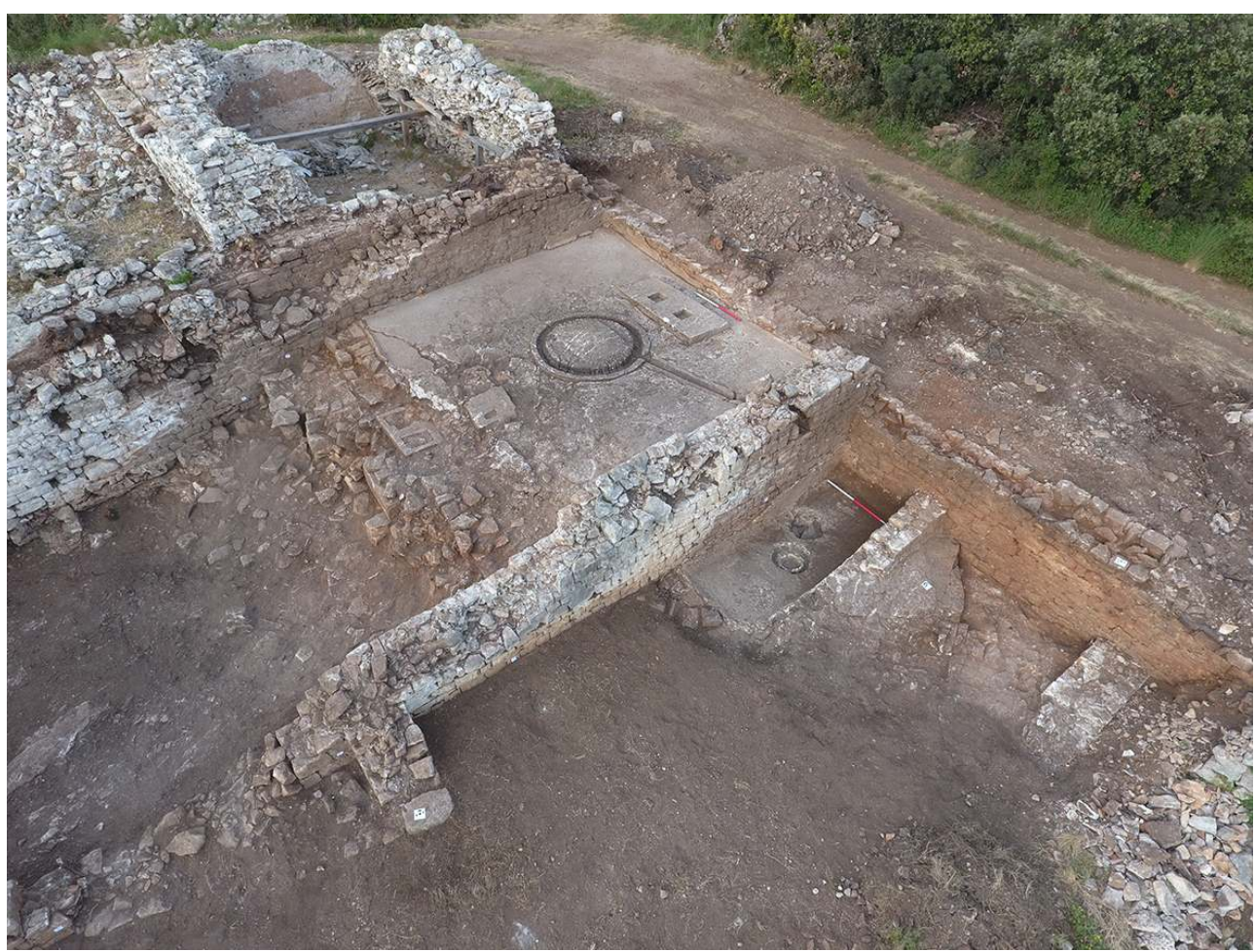

Cl. E. Botte. 
Fig. 22 - Vue depuis l'ouest, au centre de l'image, des montants du treuil d'un pressoir d'une phase antérieure apparaissant en coupe.

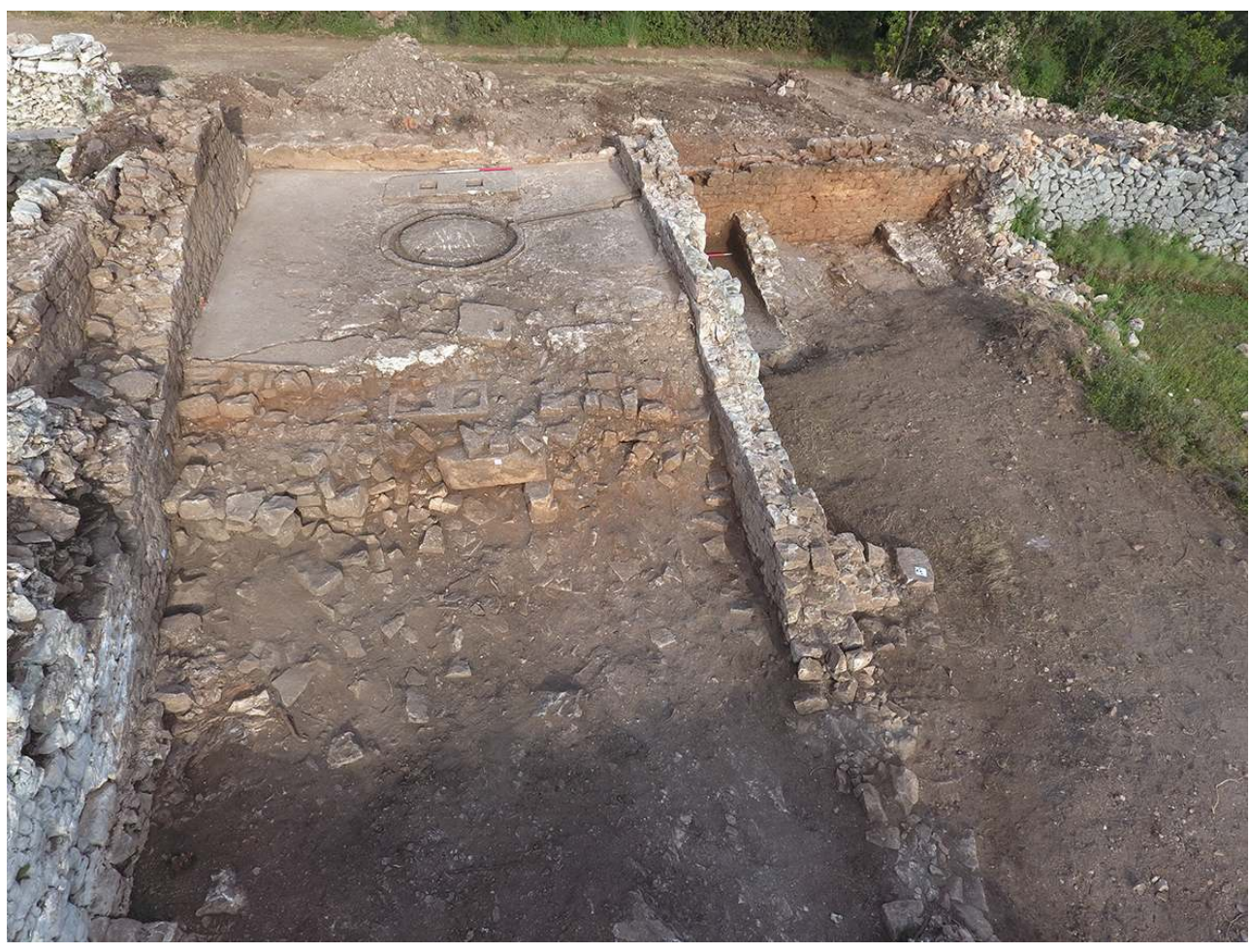

Cl. E. Botte.

Fig. 23 - Vue de détail du pressoir et du bassin de récolte situé en contrebas.

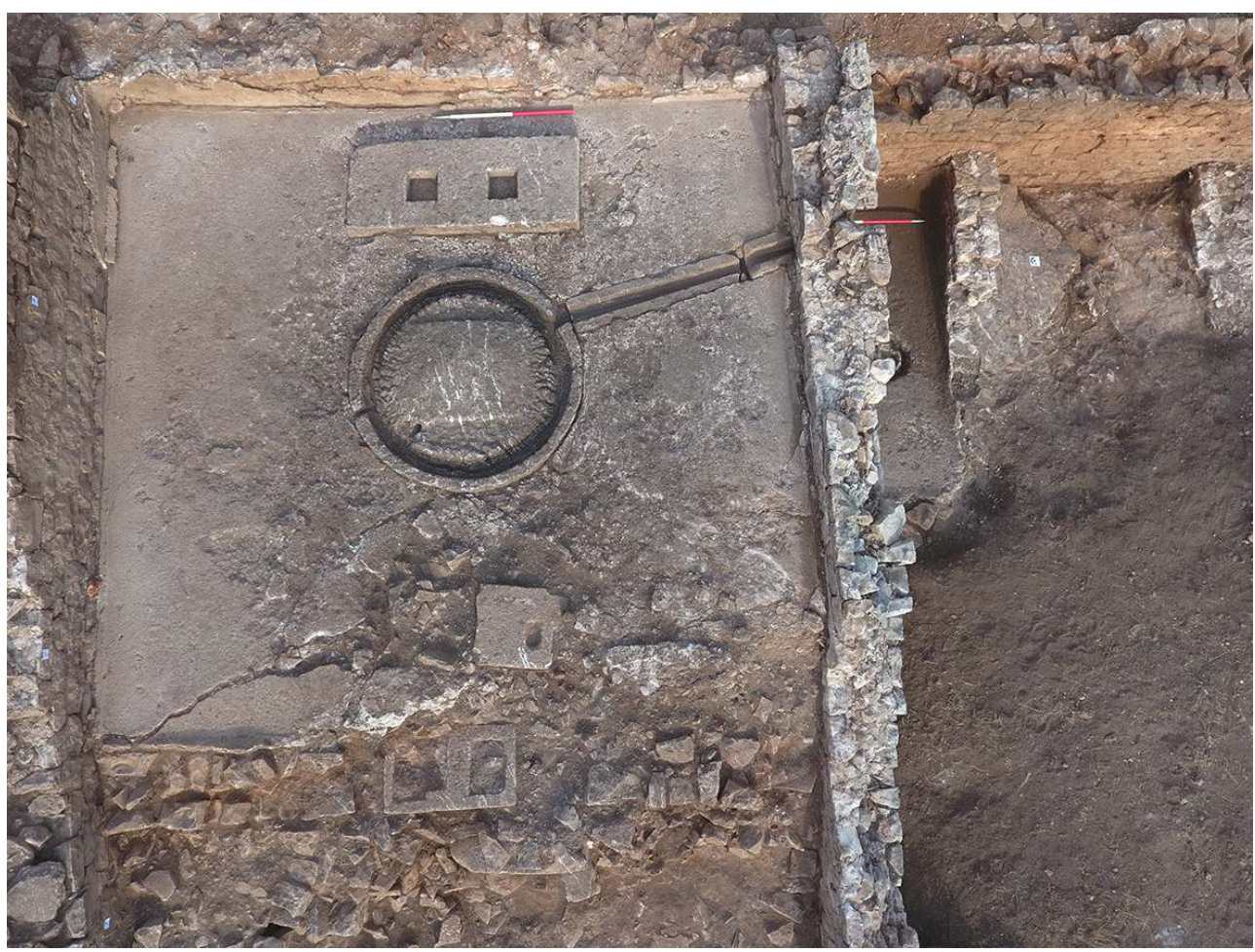

Cl. E. Botte. 
En contrebas du pressoir se trouvait un bassin (BS 8045) qui recevait le jus de la presse par le biais d'un canal aménagé directement dans le bloc de la maie et dans un grand bloc de calcaire (fig. 24). Cette canalisation (CN 8067) traversait le mur MR 8008.

Fig. 24 - Vue depuis le sud du remplissage du bassin BS 8045, récoltant le vin ou l'huile provenant du pressoir installé au-dessus.

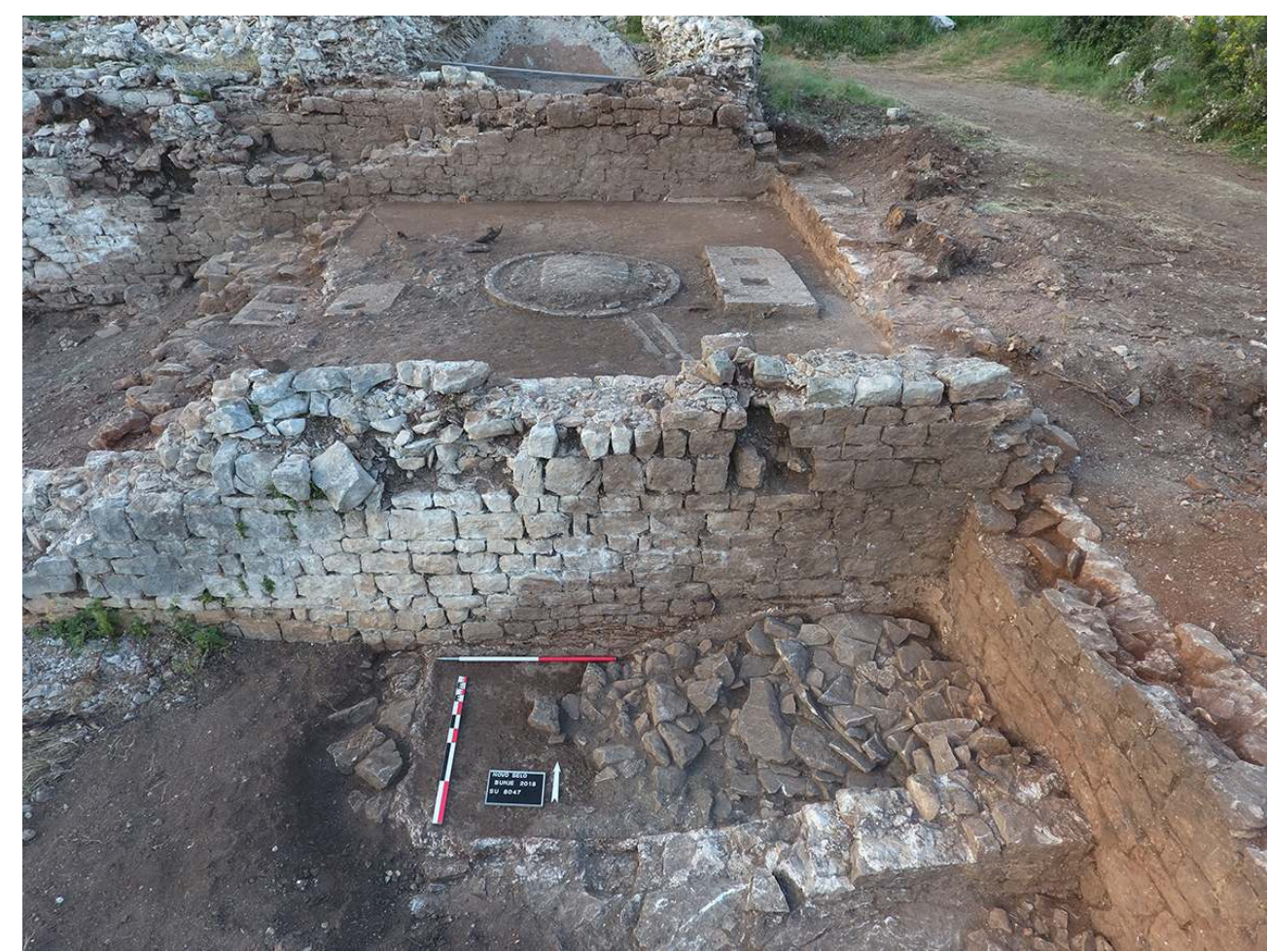

Cl. E. Botte.

Dans l'état actuel de la documentation, nous ne sommes pas en mesure de trancher clairement entre la production de vin ou d'huile dans ce pressoir. Néanmoins, d'après les autres exemples attestés en Dalmatie, nous sommes tentés de l'attribuer à la production d'huile d'olive. Nous avons pratiqué une série de prélèvements sur le bloc de la maie (8052), dans la canalisation CN 8067 et sur le sol en béton de tuileau du bassin BS 8045 que nous avons confiés à Nicolas Garnier pour que des analyses chimiques d'identification des marqueurs de résidus soient pratiquées dans son laboratoire.

\section{Secteur 9}

Durant la campagne d'octobre 2017, nous avons souhaité trouver la limite septentrionale du secteur 9, qui est concrétisée par le mur MR 9027 contre lequel vient s'appuyer le grand mur du portique donnant sur la cour centrale (fig. 25). Bien que dans un mauvais état de conservation, notre intention était premièrement d'observer dans son intégralité le mur du portique, et ensuite de déterminer si cette limite constituait également celle de la partie résidentielle de la villa, ce qui semble bien être le cas. Il faudra désormais observer durant la campagne d'octobre 2018 si ce mur se prolonge vers l'est pour clôturer la cour centrale. 
Fig. 25 - Vue depuis le sud du mur MR 9027 fermant l'espace résidentiel et sans doute la villa dans sa partie septentrionale.

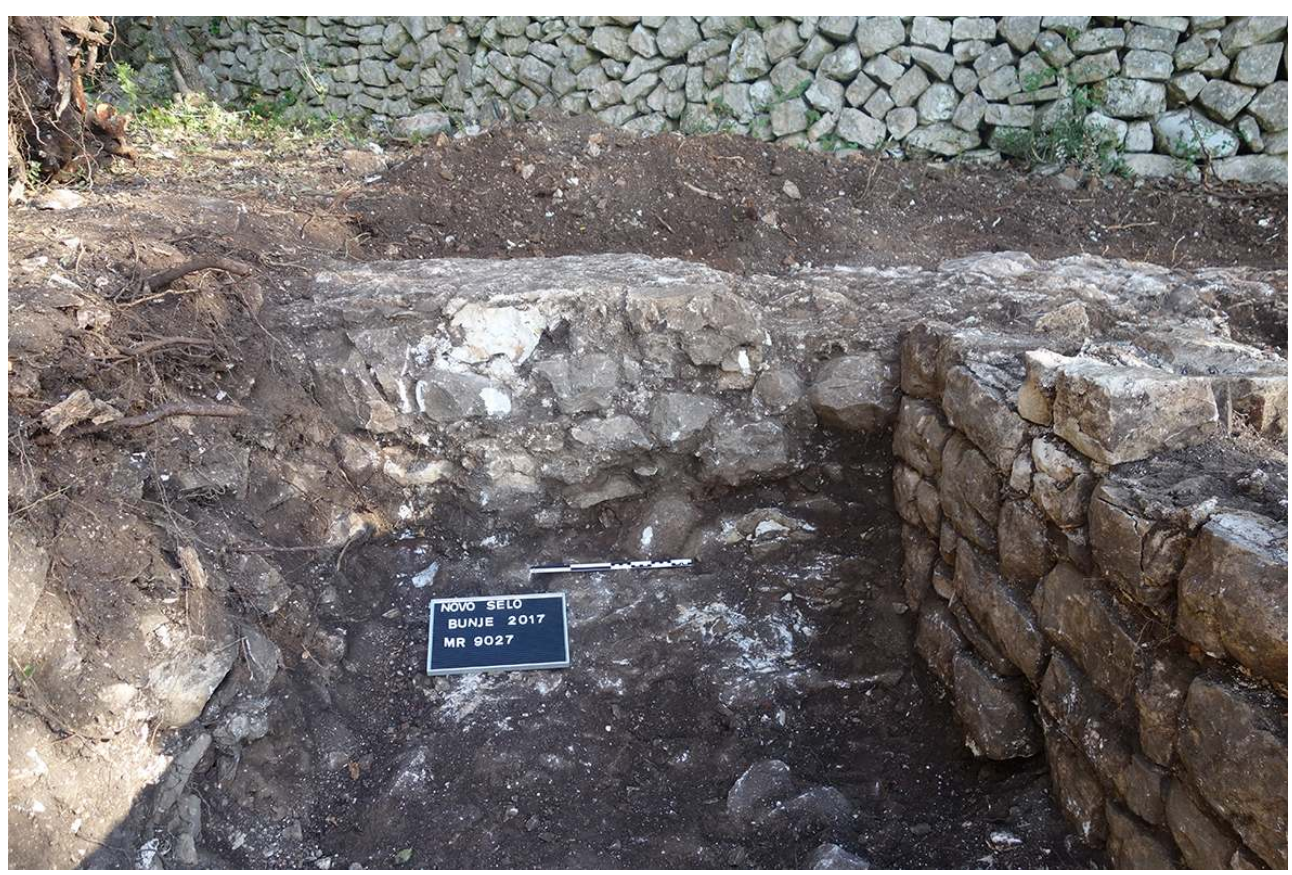

Cl. E. Botte.

\section{Mobilier}

Comme à chaque campagne, le mobilier est lavé, étudié et les éléments représentatifs dessinés avant la fin du chantier (fig. 26-29). Les mobiliers en verre, en métal, et le mobilier céramique le plus représentatif est transporté à Zagreb, tandis que le reste du matériel est entreposé dans des locaux mis à disposition par la mairie de Selca. Le profil chronologique offert par le mobilier découvert durant ces deux campagnes n'est pas différent des campagnes précédentes c'est-à-dire qu'il illustre les principales phases d'occupation des $\mathrm{II}^{\mathrm{e}}-\mathrm{III}{ }^{\mathrm{e}}$ siècles puis du $\mathrm{V}^{\mathrm{e}}$ siècle. La nouveauté réside dans le fait que pour la première fois nous avons trouvé les niveaux de la première phase d'occupation du site, datée de la fin du Ir siècle de notre ère. 
Fig. 26 - Mobilier céramique découvert durant la campagne d'octobre 2017.

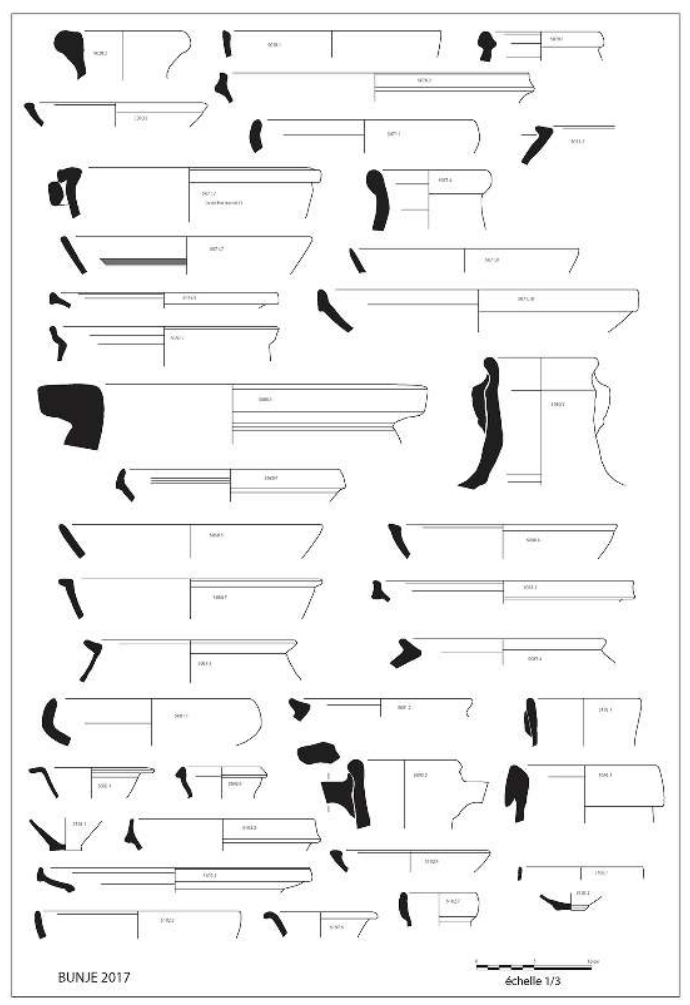

DAO A. Boisson.

Fig. 27 - Mobilier céramique du secteur 3 découvert durant la campagne d'avril 2018.

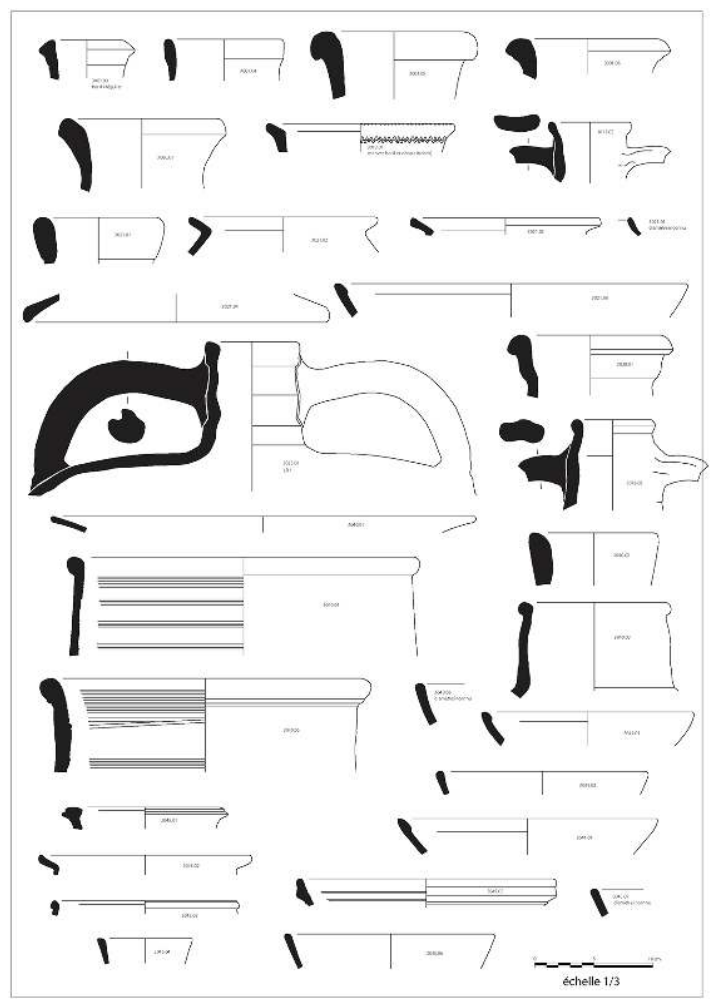

DAO A. Boisson. 
Fig. 28 - Mobilier céramique du secteur 5 découvert durant la campagne d'avril 2018.

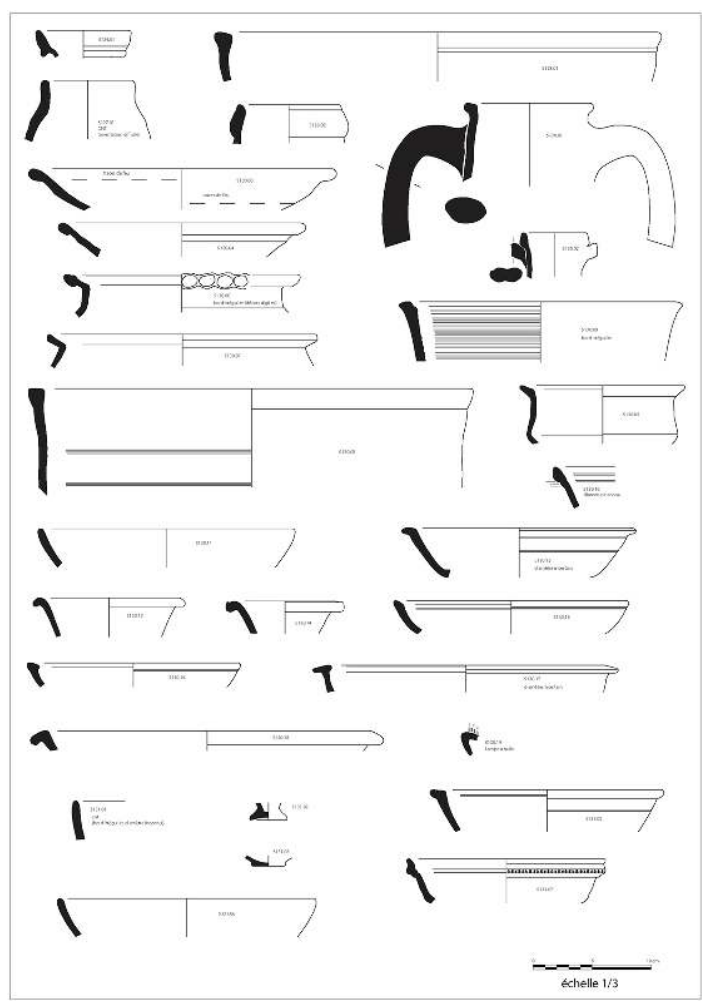

DAO A. Boisson.

Fig. 29 - Mobilier céramique des secteurs 6 et 8 découvert durant la campagne d'avril 2018.

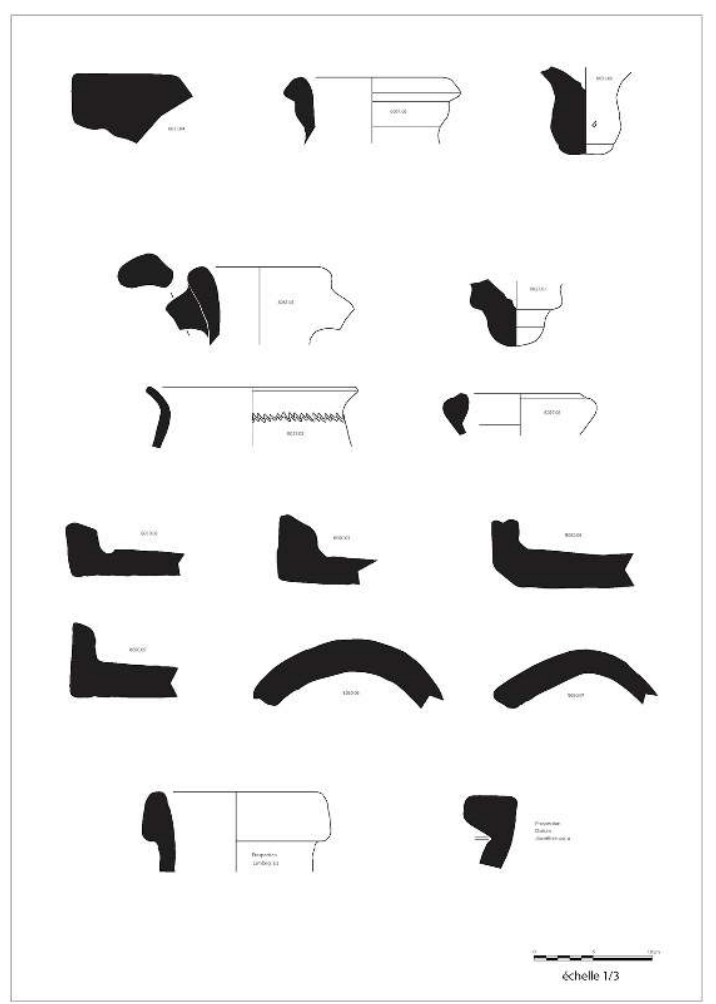

DAO A. Boisson. 


\section{Synthèse et programme des prochaines campagnes}

31 Au terme de la campagne d'avril 2018, nos connaissances sur la villa romaine de Bunje et sur ses installations ont fortement progressé (fig. 30-31). D'après les structures que nous avons dégagées et étudiées, et le mobilier qui leur était associé, il en ressort que le site est d'abord caractérisé par une petite installation, que l'on pourrait peut-être qualifier de ferme, construite durant l'époque flavienne. Dès les débuts de l'occupation, on y produit $\mathrm{du}$ vin, sans doute dans des quantités modestes mais qui doivent permettre un peu de vente de surplus. Bien que ce ne soit pas démontrable en l'état actuel de la documentation, il est probable que des oliviers aient été plantés dès la première phase d'occupation du site, mais leur rendement n'étant pas visible avant une quinzaine d'années, c'est la seconde génération qui a dû en voir le plein rendement.

Fig. 30 - Plan d'ensemble des vestiges de la villa romaine de Bunje à la fin de la campagne d'avrilmai 2018.

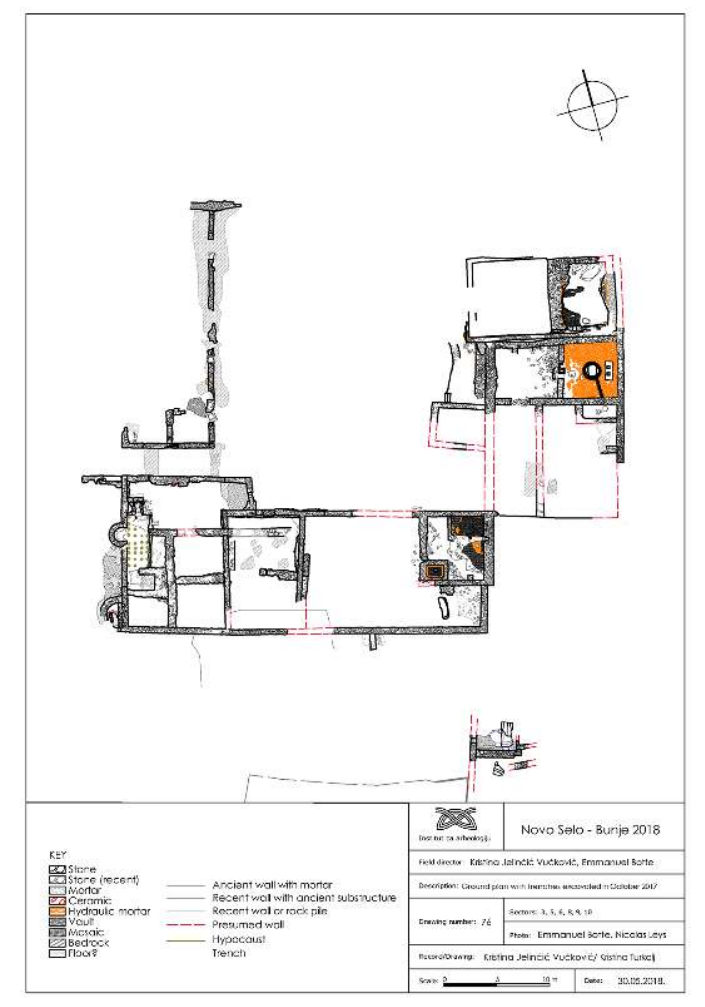

K. Jelinčić 
Fig. 31 - Vue d'ensemble de la villa romaine de Bunje à la fin de la campagne d'avril-mai 2018.

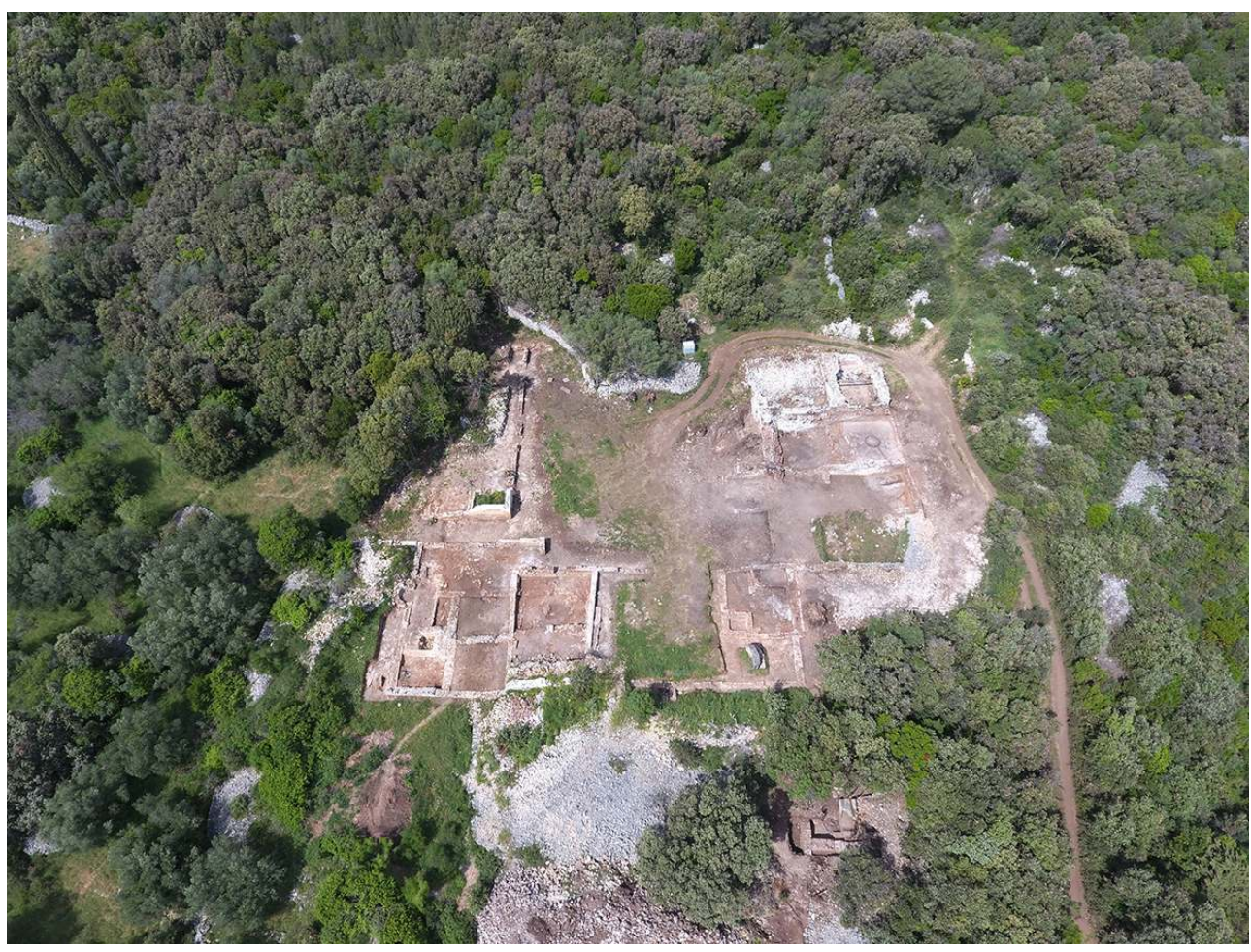

Cl. E. Botte.

32 Dans une phase que l'on peut dater du deuxième tiers du $\mathrm{II}^{\mathrm{e}}$ siècle de notre ère, donc deux générations après la première installation, un grand remaniement du site est opéré. On assiste alors au passage d'une ferme à une grande villa de production. Ce phénomène est observable aussi bien dans la pars urbana que dans la pars rustica. Dans cette dernière, la surface des fouloirs est doublée, et des équipements de production d'huile d'olive sont ajoutés, ce qui illustre une augmentation de la capacité de production du domaine. Du côté de la pars urbana de la villa, des thermes sont aménagés dans la partie méridionale et un espace sans doute réservé exclusivement à la fonction résidentielle est aménagé au nord des thermes.

33 D'après le mobilier découvert depuis le début des fouilles sur ce site, il semble que la villa soit abandonnée - et nous ne savons dire aujourd'hui si l'abandon est partiel ou total à cette période - durant le $\mathrm{III}^{\mathrm{e}}$ siècle, pour voir une réoccupation intense du site à partir du $\mathrm{V}^{\mathrm{e}}$ siècle de notre ère, avec une communauté aux croyances religieuses différentes comme l'attestent les sarcophages portant des monogrammes chrétiens découverts au nord et au sud de la villa.

34 Les objectifs des prochaines campagnes visent à combler certaines lacunes que nous devons comprendre avant de pouvoir aborder la publication de cette villa. Nous allons notamment terminer l'exploration du secteur 8 dans la pars rustica, pour déterminer s'il existait un chai destiné à la vinification et au stockage du vin. Nous allons également pratiquer un sondage dans la partie septentrionale de la villa, pour déterminer si des bâtiments existaient et si la cour était complètement fermée de ce côté ou s'il existait une ouverture permettant notamment d'accéder au bassin de rétention d'eau situé sur le flanc de la colline qui domine le site. 


\section{BIBLIOGRAPHIE}

Botte - Bertrand - Jelinčić 2016 = Bunje (Novo Selo, Croatie), dans Chronique des activités archéologiques de l'École française de Rome [en ligne], Balkans, mis en ligne le 10 mars 2016. URL : http://cefr.revues.org/1519; DOI : 10.4000/cefr.1519

Brun 1986 = J.-P. Brun, L'oléiculture antique en Provence $:$ les huileries du département du Var, Paris, 1986 (Revue archéologique de Narbonnaise, Supplément 15).

Brun 2003 = J.-P. Brun, Le vin et l'huile dans la Méditerranée antique. Viticulture, oléiculture et procédés de fabrication, Paris, 2003.

Brun 2004a = J.-P. Brun, Archéologie du vin et de l'huile de la préhistoire à l'époque hellénistique, Paris, 2004.

Brun 2004b = J.-P. Brun, Archéologie du vin et de l'huile dans l'empire romain, Paris, 2004.

Bulić 1914 = F. Bulić, Trovamenti antichi a Selca, dans Bullettino di archeologia e storia Dalmata, 37, 1914, p. 105-106.

Fisković 1981 = I. Fisković, Ranokršćanski spomenici s otoka Brača, dans Vjesnik za arheologiju $i$ historiju dalmatinsku, 78, 1981, p. 105-137.

Jelinčić 2005 = K. Jelinčić, Topografija rustičnih vila na otoku Braču, Magistarski Rad, Sveučiliste u Zagrebu, Zagreb, 2005.

Jelinčić 2012 = K. Jelinčić, Izvješće arheološkom rekognosciranju i snimanju lokaliteta Novo Selo Bunje (18.-19. travnja 2012.), Zagreb, 2012.

Kilic-Matic 2004 = A. Kilic-Matic, Prilog proučavanju tehnika i struktura gradnje rimskih vila rustika na obali rimske provincije Dalmacije = A contribution to the study of building techniques and structures at Roman villae rusticae on the coast of the Roman province of Dalmatia, dans Opuscula Archaeologica, 28, 2004, p. 91-109.

Matijasic 1983 = R. Matijasic, Cronografia dei bolli laterizi della figulina pansiana nelle regioni adriatiche , dans MEFRA, 95-2, 1983, p. 961-995.

Stančič et al. 1999 = Z. Stančič, N. Vujnovic, B. Kirigin, S. Cače, T. Podobnikar et J. Burmaz, Adriatic Islands Project, vol 2. The archaeological heritage of the island of Brač, Croatia, Oxford, 1999 (BAR International Series, 803).

Starac 2008 = A. Starac, Dragonera. Due ville romane, dans R. Auriemma, S. Karinja (dir.), Terre di Mare: l'archeologia dei paesaggi costieri e le variazioni climatiche (Trieste, 2007), Trieste, 2008, p. 304-309.

Starac 2010 = A. Starac (dir.), Dragonera, Dva Bisera - Dragonera, Two pearls, Arheolosk, Pula, 2010 ( Monografije i Katalozi, 19).

Vrsalović 1960 = D. Vrsalović, Spomenici otoka Brača, dans Brački zbornik, 4, 1960, p. 33-161.

\section{NOTES}

1. Jelinčić 2012. 
2. Fisković 1981.

3. Vrsalović 1960 , p. 62 ; Jelinčić 2005 , p. 83.

4. Bulić 1914.

5. Nous remercions L. Callegarin (Casa de Velázquez) pour son aide dans l'identification de cet objet. On peut lire sur l'avers TRAIANUS AVG-COS IIII PP ; visage regardant vers la droite, une branche de palmier incisée devant lui. Au revers : GLORIA RO-[MANORVM] ; représentation d'un trophée avec un captif assis sur le côté gauche.

INDEX

Index géographique : Bunje

Mots-clés : Dalmatie, villa romaine, Antiquité, production

institutions Avec le soutien financier de l'École française de Rome, du ministère de l'Europe et des Affaires étrangères, de l'Institut archéologique de Zagreb, du Centre Camille Jullian, de l'université Paris-Est Marne et de la mairie de Selca.

\section{AUTEURS}

\section{EMMANUEL BOTTE}

Aix Marseille Univ, CNRS, CCJ, Aix-en-Provence, France

\section{AUDREY BERTRAND}

Université Paris Est Marne-la-Vallée

\section{KRISTINA JELINČIĆ}

Institut archéologique de Zagreb

\section{NICOLAS LEYS}

Sorbonne Université

\section{ANTOINE BOISSON}

Université Paul Valéry III, Montpellier 